“( 2014 IEEE. Personal use of this material is permitted. Permission from IEEE must be obtained for all other uses, in any current or future media, including

reprinting/republishing this material for advertising or promotional purposes, creating new collective works, for resale or redistribution to servers or lists, or reuse of any copyrighted component of this work in other works." 


\title{
Contract \& Expand: I/O Efficient SCCs Computing
}

\author{
Zhiwei Zhang ${ }^{1}$, Lu Qin ${ }^{3}$, Jeffrey Xu Yu ${ }^{1,2}$ \\ ${ }^{1}$ The Chinese University of Hong Kong, Hong Kong, China \\ ${ }^{2}$ Key Laboratory of High Confidence Software Technologies Ministry of Education (CUHK Sub-Lab) \\ 1 \{zwzhang,yu\}@se.cuhk.edu.hk \\ ${ }^{3}$ Centre for Quantum Computation and Intelligent Systems, University of Technology, Sydney, Australia \\ Lu.Qin@uts.edu.au
}

\begin{abstract}
As an important branch of big data processing, big graph processing is becoming increasingly popular in recent years. Strongly connected component (SCC) computation is a fundamental graph operation on directed graphs, where an SCC is a maximal subgraph $S$ of a directed graph $G$ in which every pair of nodes is reachable from each other in $S$. By contracting each SCC into a node, a large general directed graph can be represented by a small directed acyclic graph (DAG). In the literature, there are $\mathrm{I} / \mathrm{O}$ efficient semi-external algorithms to compute all SCCs of a graph $G$, by assuming that all nodes of a graph $G$ can fit in the main memory. However, many real graphs are large and even the nodes cannot reside entirely in the main memory. In this paper, we study new I/O efficient external algorithms to find all SCCs for a directed graph $G$ whose nodes cannot fit entirely in the main memory. To overcome the deficiency of the existing external graph contraction based approach that usually cannot stop in finite iterations, and the external $D F S$ based approach that will generate a large number of random I/Os, we explore a new contraction-expansion based approach. In the graph contraction phase, instead of contracting the whole graph as the contraction based approach, we only contract the nodes of a graph, which are much more selective. The contraction phase stops when all nodes of the graph can fit in the main memory, such that the semi-external algorithm can be used in SCC computation. In the graph expansion phase, as the graph is expanded in the reverse order as it is contracted, the SCCs of all nodes in the graph are computed. Both graph contraction phase and graph expansion phase use only $\mathrm{I} / \mathrm{O}$ efficient sequential scans and external sorts of nodes/edges in the graph. Our algorithm leverages the efficiency of the semi-external SCC computation algorithm and usually stops in a small number of iterations. We further optimize our approach by reducing the size of nodes and edges of the contracted graph in each iteration. We conduct extensive experimental studies using both real and synthetic webscale graphs to confirm the $\mathrm{I} / \mathrm{O}$ efficiency of our approaches.
\end{abstract}

\section{INTRODUCTION}

Graph is an important data structure to model complex relationships among entities. A road network, a social network, and the entire WWW can be modelled as graphs, and all such graphs are huge. In this paper, we study the problem of strongly connected component (SCC) computation, which is a fundamental graph operation on directed graphs. Here, an SCC is a maximal subgraph $S$ for a given directed graph $G$, such that for every pair of nodes $u$ and $v$ in $S$, there is a directed path from $u$ to $v$ in $S$ and there is also a directed path from $v$ to $u$ in $S$.

Computing SCCs on large graphs is highly demanded by many real applications that need topological sort, reachability query processing, and graph pattern matching in graph processing. (1) Topological sort is widely used in many applications especially in planning and scheduling. In a topological sort, nodes in a directed graph are ranked according to a partial order specified by the edges. If there are cycles in the graph, all nodes in a cycle are considered as equal rank and are merged into one node. This is done by finding all SCCs in the graph. In [16], Hellings et al. propose an efficient algorithm for external bisimulation on graphs, where all nodes are assumed to be in the reverse topological order and stored on disk. This needs to find all SCCs in a preprocessing step. (2) Reachability query is a widely studied query to ask whether a node $u$ can reach another node $v$ through a directed path in a directed graph. There are many applications in social networks, biological networks, software analysis, and semantic web. Because two nodes in an SCC are reachable from each other, in the literature, almost all algorithms to process reachability queries over a general directed graph $G$ first convert $G$ into a directed acyclic graph (DAG) by contracting an SCC into a node, which needs to find all SCCs in a prepossessing step, such as [25]. (3) Pattern matching in XML data and graph data has been widely studied. Computing SCCs is an optimization technique to compress a large graph for processing pattern matching queries [15].

In the literature, there are efficient in-memory and $\mathrm{I} / \mathrm{O}$ efficient semi-external algorithms to compute all SCCs of a directed graph $G$. An in-memory algorithm requires $G$ to reside entirely in memory and a semi-external algorithm requires all nodes of $G$ to reside entirely in memory. For the in-memory algorithm, the Kosaraju-Sharir algorithm [3] can find all SCCs for a directed graph in linear time w.r.t. the size of the graph, by depth first searching the graph twice in memory. For the semi-external algorithm, Zhang et al. [26] propose an I/O efficient algorithm to compute all SCCs by constructing a special in-memory spanning tree of $G$ using sequential scans of the graph on disk. However, due to the fact that the sizes of many real large graphs keep growing rapidly, even the nodes of a graph cannot reside entirely in the main memory. For example, the social network graph in Facebook contains 1.11 billion active nodes and more than 150 billion edges. ${ }^{1}$ As a small part of the entire web graph, WEBSPAM-

\footnotetext{
${ }^{1}$ http://newsroom.fb.com/
} 
UK2007 ${ }^{2}$ contains $105,896,555$ pages and 4 billion edges in 114,529 hosts in the .UK domain in May 2007.

In order to handle a graph $G(V, E)$ where $V$ cannot fit entirely in memory $M$, a naive way to externalize the inmemory DFS requires $O(|E|)$ I/Os. Chiang et al. [10] propose an algorithm with I/O complexity $O\left(|V|+\frac{|V|}{M} \cdot \operatorname{scan}(|E|)+\right.$ $\operatorname{sort}(|E|))$. Later, Kumar and Schwabe [17] and Buchsbaum et al. [8] improve the I/O complexity to $O\left(\left(|V|+\frac{|E|}{B}\right) \cdot \log _{2} \frac{|V|}{B}\right.$ $+\operatorname{sort}(|E|))$ by maintaining the nodes that should not be traversed using tournament trees [17] and buffered repository trees [8] respectively, where $B$ is the disk block size. Despite their theoretical guarantees, these algorithms are considered impractical for general directed graphs that encountered in real applications, due to the large number of random I/Os generated. Cosgaya-Lozano et al. [13] study a heuristic external algorithm to compute all SCCs for a directed algorithm based on contraction used by Chiang et al. [10] which is for undirected graphs. But, for directed graphs, the algorithm may end up an infinite loop and cannot compute all SCCs.

In this paper, we study external algorithms for SCC computation. The main contributions of this work are summarized below. Firstly, we analyze the deficiency of the existing DFS based algorithm [8] that consumes a large number of random I/Os and the contraction based algorithm [13] that needs large number of iterations and may end up an infinite loop. Secondly, we propose a new two-phase algorithm with graph contraction followed by graph expansion. In graph contraction, we only contract the number of nodes of the graph with bounded number of new edges generated. We stop when all nodes can fit in the main memory and process the contracted graph using an I/O efficient semi-external algorithm. Using nodes contraction, the number of iterations can be significantly reduced comparing to [13]. In graph expansion, the removed nodes are put back into the graph in a reverse order of their removal, while the SCCs of all nodes are computed. We analyze the I/O cost of our approach and show that our algorithm can significantly reduce the number of random I/Os comparing to [8]. Thirdly, we introduce techniques to further reduce the $\mathrm{I} / \mathrm{O}$ cost of our algorithm by reducing the number of nodes and edges generated in each iteration of graph contraction. Finally, we conduct extensive experimental studies using both real and synthetic web-scale graphs to confirm the I/O efficiency of our approaches.

The remainder of this paper is organized as follows. In Section II, we discuss the preliminaries and give the problem statement for computing SCCs. In Section III, we discuss existing solutions on both external and semi-external algorithms for computing SCCs. In Section IV, we analyze the deficiency of existing external approaches and outline our two-phase approach with graph contraction followed by graph expansion. We introduce the graph contraction phase in Section $\mathrm{V}$ and discuss the graph expansion phase in Section VI. We study optimization techniques to further reduce the I/O cost of our algorithm in Section VII. In Section VIII, we report our

\footnotetext{
${ }^{2}$ http://barcelona.research.yahoo.net/webspam/datasets/uk2007/
}

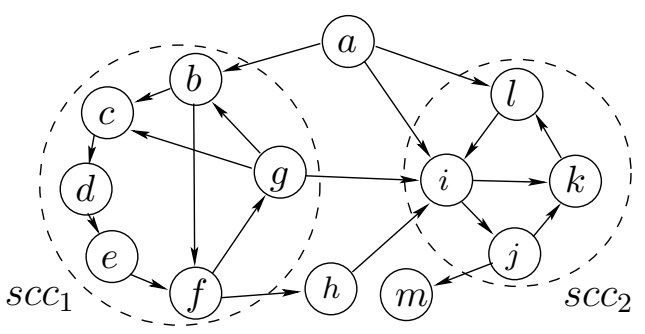

Fig. 1. A Graph $G$ with 2 SCCs

experimental results. We discuss the related work in Section IX and conclude the paper in Section X.

\section{PRoblem Definition}

We model a directed graph as $G(V, E)$, where $V(G)$ represents the set of nodes and $E(G)$ represents the set of directed edges in $G$. For simplicity, we also use $V$ and $E$ to denote $V(G)$ and $E(G)$ respectively, when it is obvious. Each node $v \in V(G)$ has a unique identity in $G$, denoted by $\operatorname{id}(v)$, which specifies a unique total order among all nodes in $G$.

For each node $v \in V(G)$, we use $\operatorname{nbr}_{i n}(v, G)$ to denote the set of in-neighbors of $v$ in $G$, and we use $\operatorname{nbr}_{\text {out }}(v, G)$ to denote the set of out-neighbors of $v$ in $G$. We use $\operatorname{nbr}(v, G)$ to denote the set of in-neighbors and out-neighbors of $v$ in graph $G$. We have $\operatorname{nbr}_{i n}(v, G)=\{u \mid(u, v) \in E(G)\}$, $\operatorname{nbr}_{\text {out }}(v, G)=\{u \mid(v, u) \in E(G)\}$ and $\operatorname{nbr}(v, G)=$ $\operatorname{nbr}_{\text {in }}(v, G) \cup \operatorname{nbr}_{\text {out }}(v, G)$. We use $\operatorname{deg}_{\text {in }}(v, G), \operatorname{deg}_{\text {out }}(v, G)$, and $\operatorname{deg}(v, G)$ to denote the in-degree, out-degree and total degree of a node $v \in V(G)$ respectively. We have $\operatorname{deg}_{\text {in }}(v, G)=\left|\operatorname{nbr}_{\text {in }}(v, G)\right|, \operatorname{deg}_{\text {out }}(v, G)=\left|\operatorname{nbr}_{\text {out }}(v, G)\right|$ and $\operatorname{deg}(v, G)=|\operatorname{nbr}(v, G)|$.

Given a graph $G(V, E)$, a vertex cover of $G$ is a subset $V^{\prime} \subseteq V$ such that if $(u, v) \in E$, then either $u \in V^{\prime}$ or $v \in V^{\prime}$. In other words, each vertex covers its incident edges and a vertex cover of $G$ is a set of vertices that covers all $E(G)$ [12]. It is trivial that any superset for a vertex cover is still a vertex cover. A minimum vertex cover is a vertex that has the minimum set cardinality among all the vertex covers of $G$.

Given a graph $G$, a path $p=\left(v_{1}, v_{2}, \cdots, v_{k}\right)$ is a sequence of $k$ nodes in $V$ such that, for each $v_{i}(1 \leq i<k),\left(v_{i}, v_{i+1}\right) \in$ $E$. A node $v_{i}$ can reach a node $v_{j}$ in $G$, denoted $v_{i} \rightarrow v_{j}$, iff there exists a path from $v_{i}$ to $v_{j}$ in $G$. A node $v_{i}$ cannot reach a node $v_{j}$ in $G$, denoted $v_{i} \nrightarrow v_{j}$, iff there exists no path from $v_{i}$ to $v_{j}$ in $G$. A node $v_{i}$ is strongly connected to a node $v_{j}$, denoted as $v_{i} \leftrightarrow v_{j}$, iff $v_{i} \rightarrow v_{j}$ and $v_{j} \rightarrow v_{i}$ in $G$. Here, $\leftrightarrow$ is an equivalence relation, which is reflexive, symmetric, and transitive. We use $v_{i} \leftrightarrow v_{j}$ to denote that node $v_{i}$ is not strongly connected to node $v_{j}$. A strongly connected component (SCC) of $G$ is the maximal set of nodes $V_{s}(\subseteq V)$ such that, for every pair of nodes $v_{i}$ and $v_{j}$ in $V_{s}, v_{i} \leftrightarrow v_{j}$, and for every pair of nodes $v_{i}$ and $v_{k}$ where $v_{i} \in V_{s}$ and $v_{k} \notin V_{s}$, $v_{i} \leftrightarrow v_{k}$. Each SCC of $G$ has a unique identity. For each node $v \in V(G)$, we use $\operatorname{SCC}(v, G)$ to denote the SCC that $v$ belongs to. For any two nodes $u \in V(G)$ and $v \in V(G)$, $u \leftrightarrow v \Leftrightarrow \operatorname{SCC}(u, G)=\operatorname{SCC}(v, G)$. For any set of nodes $S \subseteq V(G)$, we use $\operatorname{SCC}(S, G)$ to denote the set of SCCs that 


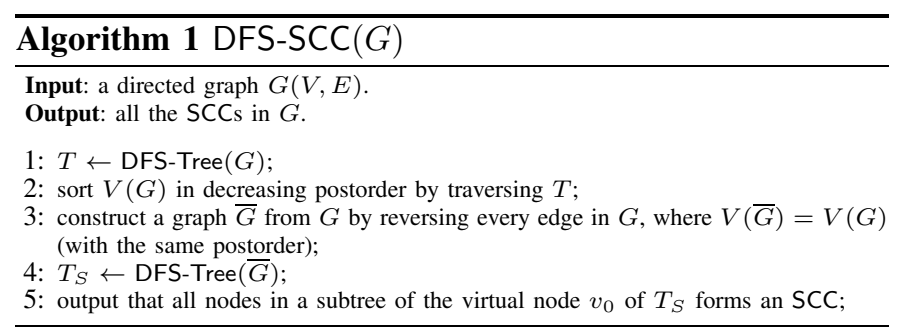

nodes in $S$ belong to, i.e., $\operatorname{SCC}(S, G)=\{\operatorname{SCC}(v, G) \mid v \in S\}$.

Example 2.1: Fig. 1 shows a graph $G$ with 13 nodes and 20 edges. For nodes $b$ and $e, b \leftrightarrow e$ since $b \rightarrow e$ through path $(b, c, d, e)$ and $e \rightarrow b$ through path $(e, f, g, b)$. There are 2 SCCs, $S C C_{1}$ and $S C C_{2}$, where $S C C_{1}=\{b, c, d, e, f, g\}$ and $S C C_{2}=\{i, j, k, l\}$.

In this paper, we assume that the graph $G$ cannot reside entirely in the main memory. For all I/O operations, we follow the standard I/O model in [2]. We use $M$ to denote the size of the main memory and use $B$ to denote the size of each block on disk. All data are read/written in blocks. We assume $M \geq 2 \times B$. We use $\operatorname{sort}(m)$ to denote the I/O cost of external sorting $m$ elements on disk and we use $\operatorname{scan}(m)$ to denote the I/O cost of sequentially scanning all $m$ elements once on disk. In the I/O model [2], we have $\operatorname{scan}(m)=\Theta\left(\frac{m}{B}\right)$, and $\operatorname{sort}(m)=\Theta\left(\frac{m}{B} \cdot \log _{\frac{M}{B}} \frac{m}{B}\right)$.

Problem Statement: compute all strongly connected components (SCCs) for a large directed graph $G(V, E)$ with limited memory $M$. Here $2 \times B \leq M<\|G\|$ where $B$ is the block size and $\|G\|$ is the space for the entire graph $G$.

\section{Existing SOlutions}

In the literature, there are two external algorithms and two semi-external algorithms to compute all SCCs for a directed graph $G$. A semi-external algorithm assumes that all nodes of the graph can reside entirely in the main memory, i.e., $M \geq c \times|V|$ for a constant $c$, and an external algorithm only assumes that at least two disk blocks can fit in the main memory, i.e., $M \geq 2 \times B$. The two external algorithms are based on contraction [13] and external depth first search (DFS) [8] respectively, and the two semi-external algorithms are based on semi-external DFS [23] and a special spanning tree BR-Tree of $G$ respectively [26].

Contraction Based EM-SCC: Cosgaya-Lozano et al. [13] provide a heuristic algorithm, called EM-SCC, to compute all SCCs for a large directed graph. The idea is taken from the contraction-based algorithm to compute all connected components for an undirected graph [10]. Given limited memory $M$, EM-SCC compresses a graph iteratively by contraction until the graph can fit in $M$. In brief, it processes $G$ in iterations, $G=G_{0}, G_{1}, G_{2}, \cdots, G_{f}$. In the $i$-th iteration, EM-SCC contracts some partial SCCs into a node and compresses $G_{i}$ to be a smaller $G_{i+1}$. The last $G_{f}$ must fit in $M$. In the $i$-th iteration $(i<f), G_{i}$ cannot fit in $M$. EM-SCC partitions $G_{i}$ into smaller partitions, $G_{i_{1}}, G_{i_{2}}, \cdots$ where $G_{i_{j}}$ can fit in $M$. EM-SCC computes SCCs using an in-memory algorithm for $G_{i_{j}}$, and compresses $G_{i}$ by contracting an SCC in $G_{i_{j}}$ into a node.
Unlike the algorithm [10] which ensures an undirected graph $G$ can fit into main memory in a log number of iterations, EM-SCC cannot stop in a finite number of iterations for directed graphs in the following cases to compute all SCCs. (Case-1) An SCC of $G_{i}$ appears across a number of partitions, and the partitions cannot be further compressed by contraction. (Case-2) $G_{i}$ is a directed acyclic graph, but cannot fit in $M$. When $G$ contains a large SCC or a large number of small/mid sized SCCs, the probability of Case-1 to happen is high. When $G$ is a $D A G$-liked graph, the probability of Case-2 to happen is high. Either case happens frequently in real world graphs.

Depth First Search Based DFS-SCC: DFS-SCC computes all SCCs by simulating the in-memory Kosaraju-Sharir algorithm [3] which traverses the graph $G$ twice using depth first search. The framework of DFS-SCC is shown in Algorihtm 1. In the first time traversal, it obtains a decreasing postorder of nodes over the DFS tree obtained by DFS-Tree $(G)$ (lines 1-2). It then constructs a graph $\bar{G}$ by reversing every edge in $G$ (line 3 ). Because $V(\bar{G})=V(G)$, with the same decreasing postorder, it calls DFS-Tree $(\bar{G})$ again to obtain a new DFS tree $T_{S}$ (line 4 ). Here, all nodes in a subtree rooted at a child of the virtual node $v_{0}$ of $T_{S}$ are in the same SCC. The key operation of DFS-SCC is DFS-Tree which constructs a DFS tree for a graph $G .^{3}$ Thus, we introduce existing external DFS algorithms below.

In the literature, external DFS algorithms are proposed by Kumar et al. [17] and Buchsbaum et al. [8]. [8] is an improvement for [17]. The basic idea of [8] is to simulate the internal memory DFS algorithm by maintaining the visited nodes using an augmented external $(2,4)$-tree, called buffer repository tree (BRT). The I/O complexity for DFS using the algorithm in [8] is $O\left(\left(|V|+\frac{|E|}{B}\right) \log _{2} \frac{|V|}{B}+\operatorname{sort}(|E|)\right)$. Comparing to the trivial external memory DFS which consumes $O(|E|)$ I/Os, the algorithm in [8] does not improve significantly especially on sparse graphs [5], and a large number of random I/Os are generated by the algorithm in [8]. Thus, computing SCCs based on external DFS is not I/O efficient.

Example 3.1: Consider $G$ in Fig. 1. The first DFS traverses $G$ in abcdefgijklmh, and its decreasing postorder is abcdefhgijmkl. With the decreasing postorder, in the second $D F S$, the root $v_{0}$ of the DFS tree has 5 subtrees representing 5 SCCs $\{a\},\{b, c, d, e, f, g\},\{h\},\{i, j, k, l\}$, and $\{m\}$.

Semi-External Approach Semi-SCC: Since there is no efficient external algorithm to compute all SCCs for a graph $G$ in the literature, some papers focus on semi-external algorithms by relaxing the condition $2 \times B \leq M<\|G\|$ to $c \times|V| \leq M<\|G\|$, where $c$ is a constant. Following the framework in Algorithm 1, a semi-external algorithm can be designed by applying semi-external DFS [23] when generating the DFS tree (line 1 and line 4). In [23], given a graph $G$, a spanning tree $T$ of $G$ is maintained in memory using $O(|V|)$ space. The algorithm iteratively scans the edges of $G$ on disk and updates $T$ until $T$ becomes a DFS tree. The semi-external

\footnotetext{
${ }^{3}$ We do not need to construct a DFS tree explicitly. We only need to obtain the DFS order of all nodes in the graph.
} 


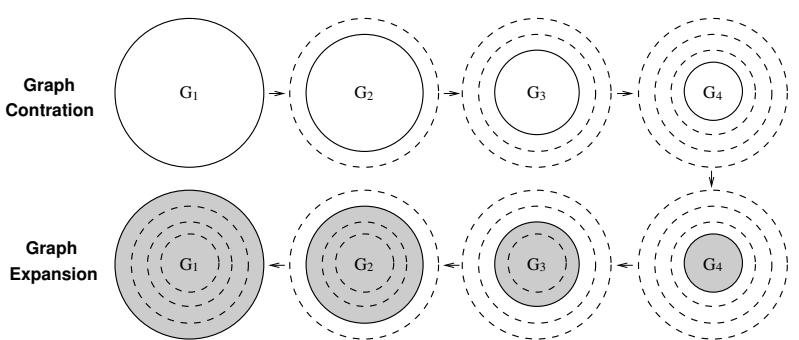

Fig. 2. A Solution Overview

DFS algorithm in [23] is much more efficient than the external DFS algorithm in [8]. However, it is not optimized for SCC computation using the Kosaraju-Sharir algorithm (Algorithm 1). This is because Algorithm 1 needs to maintain a total order (decreasing postorder) of nodes in the first DFS (line 1-2), to be used in the second DFS (line 4). As a result, in the first $D F S$, nodes cannot be contracted or removed even if partial SCCs have been found.

In order to improve the semi-external DFS based approach using Algorithm 1, Zhang et al. [26] develop a more efficient semi-external algorithm to compute all SCCs, by defining a weaker order based on the depth of a node in a spanning tree (BR-Tree) of $G$. Using the weaker order, only one BR-Tree needs to be constructed in memory. The algorithm iteratively scans the edges of $G$ on disk and updates the BR-Tree until no new SCC can be found. When updating the BR-Tree, each partial SCC can be contracted into one node, and nodes that will not contribute to any new SCCs can be removed from $G$. The I/O cost to compute all SCCs is largely reduced.

Although Semi-SCC is much more efficient than external $D F S$ and external contraction based approaches, it assumes that $M \geq c \times|V|$. In this paper, we aim to design an efficient external algorithm to compute SCCs when $M<c \times|V|$.

\section{A NEW CONTRACTION-EXPANSION APPROACH}

Given that the contraction based EM-SCC may end up infinite iterations, and the DFS based DFS-SCC can generate a large number of random I/Os. In this paper, we propose a novel contraction-expansion based external algorithm to compute all SCCs for a graph $G$. Instead of accessing each node one by one in EM-SCC, our algorithm computes the SCCs of all nodes in batches in order to reduce the number of random I/Os used to access each node. Our algorithm is processed in two phases, namely, graph contraction and graph expansion.

In the graph contraction phase, a list of graphs $G_{1}, G_{2}, \cdots$, $G_{l}$ are generated, where $G_{1}=G$, and for each $1 \leq i<l$, $G_{i+1}$ is generated by removing a batch of nodes from $G_{i}$, i.e., $V\left(G_{i+1}\right) \subset V\left(G_{i}\right)$. It stops until all nodes of the graph can fit in memory, i.e., $c \times\left|V\left(G_{l}\right)\right| \leq M$ for a constant $c$. In other words, SCCs of $G_{l}$ can be computed using Semi-SCC.

In the graph expansion phase, after computing all SCCs of $G_{l}$ using Semi-SCC, the removed nodes are added back to the graph in the reverse order of their removal in the graph contraction phase, i.e., the lastly removed nodes in the graph contraction phase are firstly added back in the graph expansion phase. When a node is added back, the SCC it belongs to is computed. More specifically, given that all

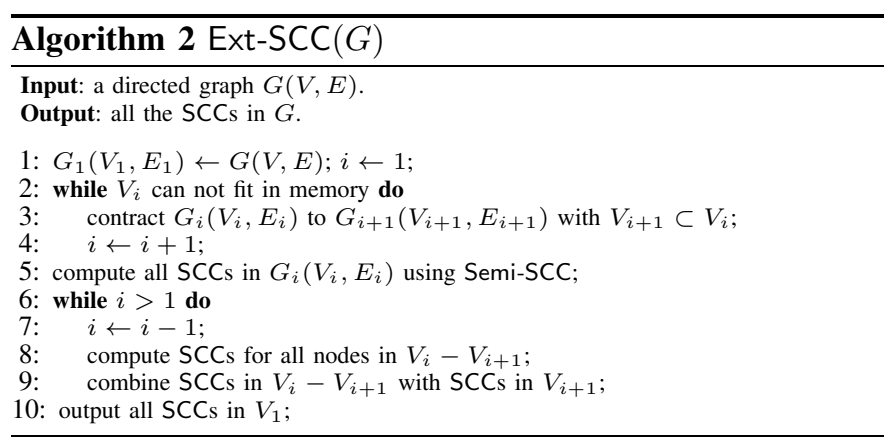

SCCs of $G_{l}$ are computed using Semi-SCC, we compute the SCCs for nodes $V\left(G_{l-1}\right)-V\left(G_{l}\right), V\left(G_{l-2}\right)-V\left(G_{l-1}\right)$, $\cdots, V\left(G_{1}\right)-V\left(G_{2}\right)$ in order. Since $V(G)=V\left(G_{1}\right)=$ $V\left(G_{l}\right) \cup\left(V\left(G_{l-1}\right)-V\left(G_{l}\right)\right) \cup\left(V\left(G_{l-2}\right)-V\left(G_{l-1}\right)\right) \cdots \cup$ $\left(V\left(G_{1}\right)-V\left(G_{2}\right)\right)$, the SCCs for all nodes are computed after graph expansion.

The two phases are illustrated in Fig. 2 where gray parts represent the nodes whose SCCs are computed. Our algorithm Ext-SCC to compute all SCCs in a graph $G$ is shown in Algorithm 2. Initially, $G_{1}=G$ and $i=1$ (line 1). The graph contraction phase is shown in line 2-4. It iteratively generates a new graph $G_{i+1}$ based on $G_{i}$ with a smaller number of nodes and stops when the nodes of the graph can fit in the main memory. The graph expansion phase is shown in line 59. It first computes all SCCs of $V\left(G_{i}\right)$ using the semi-external algorithm Semi-SCC (line 5). Then it iteratively computes all SCCs for nodes $V\left(G_{i}\right)-V\left(G_{i+1}\right)$ with decreasing order of $i$ (line 6-9). Finally, all SCCs of $V\left(G_{1}\right)$ are output since $G_{1}=G$ (line 10).

Below, we analyze the deficiencies of the existing external DFS based DFS-SCC algorithm and external contraction based EM-SCC algorithm, comparing to our Ext-SCC algorithm.

For DFS-SCC, as introduced in Section III, the I/O complexity of the algorithm is $O\left(\left(|V|+\frac{|E|}{B}\right) \log _{2} \frac{|V|}{B}+\operatorname{sort}(|E|)\right)$, which is higher than $O(|V|)$. The high $\mathrm{I} / \mathrm{O}$ cost is produced by the large number of random accesses when visiting nodes one by one on disk, and it is costly to access each node using one random disk access. In our algorithm, instead of processing nodes one by one, we process the nodes of $G$ in batches. As shown in Algorithm 2, we contract the graph $G_{i}$ by removing a batch of nodes $V\left(G_{i}\right)-V\left(G_{i+1}\right)$ with increasing $i$ in the graph contraction phase, and add the removed nodes back to compute the SCCs for each batch of removed nodes in a reverse order in the graph expansion phase. As we will show later, both the graph contraction phase and the graph expansion phase are processed using only sequential scans and external sorts. In such a way, all nodes/edges of the graph can be processed in blocks and the number of random accesses is minimized.

For EM-SCC, it is a contraction based algorithm with some critical problems as introduced in Section III. Our contractionexpansion based algorithm Ext-SCC can solve the problems. Firstly, EM-SCC may not be able to terminate. In our Ext-SCC algorithm, when generating $G_{i+1}$ from $G_{i}$, we make sure that $V\left(G_{i+1}\right)$ is a proper subset of $V\left(G_{i}\right)$ by removing a batch of nodes from $V\left(G_{i}\right)$. Thus, our algorithm can always terminate. 
Furthermore, our stop condition only requires that all nodes $V\left(G_{i}\right)$ can fit in the main memory, which is usually much smaller than the size of the whole graph $G_{i}$. Our stop condition is easier to be satisfied comparing to EM-SCC which requires the whole graph $G_{i}$ to fit in the main memory. Secondly, even if EM-SCC can terminate in a finite number of iterations, the contraction is unstable since it relies largely on the order of edges stored on disk. It is possible that only a small number of nodes are contracted in each iteration. In our ExtSCC algorithm, the selection of nodes to be removed in each iteration does not rely on the order of edges stored on disk, and thus our algorithm is much more stable than EM-SCC. As confirmed in our experiments, our algorithm can usually terminate in a small number of iterations.

In the following, we discuss the two phases, and propose techniques to further minimize the I/O cost.

\section{Graph CONTRACTION}

In this section, we introduce how to contract a graph $G_{i+1}$ from graph $G_{i}$. As introduced before, when constructing $G_{i+1}$, we should make sure that the nodes of $G_{i+1}$ is a proper subset of $G_{i}$ by removing at least one node from $G_{i}$, i.e., $V\left(G_{i+1}\right) \subset$ $V\left(G_{i}\right)$. In order to reduce the number of iterations, the number of nodes to be removed in each iteration should be as large as possible. However, the nodes to be removed in each iteration $i$ cannot be arbitrarily selected. In order to make sure that all SCC $s$ can be computed correctly, for each graph $G_{i}$, the newly contracted graph $G_{i+1}$ should satisfy the following three properties.

- (Contractible): The number of nodes in the contracted graph $G_{i+1}$ should be smaller than the number of nodes in $G_{i}$, i.e., $V\left(G_{i+1}\right) \subset V\left(G_{i}\right)$.

- (SCC-preservable): For any two nodes $u \in V\left(G_{i+1}\right)$ and $v \in V\left(G_{i+1}\right), u$ and $v$ are in the same SCC in $G_{i+1}$ iff $u$ and $v$ are in the same $\operatorname{SCC}$ in $G_{i}$, i.e., $\operatorname{SCC}\left(u, G_{i+1}\right)=$ $\operatorname{SCC}\left(v, G_{i+1}\right) \Leftrightarrow \operatorname{SCC}\left(u, G_{i}\right)=\operatorname{SCC}\left(v, G_{i}\right)$.

- (Recoverable): If a node $v \in V\left(G_{i}\right)$ is removed when constructing $G_{i+1}$ from $G_{i}$, the connectivity of $v$ to other nodes in $G_{i+1}$ can be recovered using only $v$ 's neighbors in $G_{i+1}$. In other words, if $v$ is absent in $V\left(G_{i+1}\right)$, all $v$ 's neighbors should be in $V\left(G_{i+1}\right)$, i.e., $v \in V\left(G_{i}\right)-$ $V\left(G_{i+1}\right) \Rightarrow \operatorname{nbr}\left(v, G_{i}\right) \subseteq V\left(G_{i+1}\right)$.

In the following, given a graph $G_{i}\left(V_{i}, E_{i}\right)$, we introduce how to construct the nodes $V_{i+1}$ of $G_{i+1}$ and edges $E_{i+1}$ of $G_{i+1}$ respectively. We will show that by constructing $V_{i+1}$, the contractible and recoverable properties are satisfied, and by constructing $E_{i+1}$, the SCC-preservable property is satisfied.

To construct $V_{i+1}$ : Given $G_{i}\left(V_{i}, E_{i}\right)$, we first investigate the properties of $V_{i+1}$. We start from the recoverable property, i.e., for any $v \in V_{i}-V_{i+1}, \operatorname{nbr}\left(v, G_{i}\right) \subseteq V_{i+1}$. Consider an arbitrary edge $(u, v) \in E_{i}$, if $v \notin V_{i+1}$, i.e., $v \in V_{i}-V_{i+1}$, then $u \in V_{i+1}$, since $u \in \operatorname{nbr}\left(v, G_{i}\right)$. Similarity, if $u \notin V_{i+1}$ then $v \in V_{i+1}$. In other words, for any edge $(u, v) \in E_{i}$, either $v \in V_{i+1}$ or $u \in V_{i+1}$. We have the following lemma.

Lemma 5.1: $G_{i+1}$ is recoverable if and only if $V_{i+1}$ is a vertex cover of $G_{i}$.

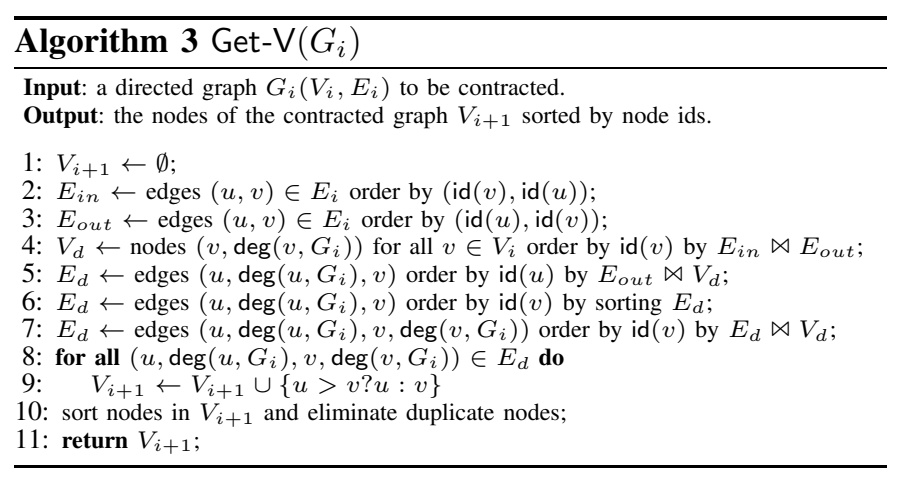

Proof Sketch: The lemma can be derived easily from the above discussion.

In order to reduce the number of iterations in the ExtSCC algorithm, the cardinality of $V_{i+1}$ should be as small as possible. This leads to the minimum vertex cover problem which is NP-hard [3]. In the literature, a lot of approximate algorithms have been developed to find the vertex cover of a graph $G$. When the graph $G$ cannot fit in the main memory, there are semi-external and external algorithms to find an approximate minimum vertex cover for a graph $G$. For the semiexternal algorithm, in [9], James et al. develop a streaming algorithm to find a vertex cover with the approximation ratio 2 , by maintaining an in-memory hash table $H$. However, since $|H|=O(|V(G)|)$ in the worst case, the algorithm cannot be used directly in constructing $V_{i+1}$. For the external algorithm, in [7], an algorithm is introduced to find a vertex cover with an approximation ratio $\frac{\sqrt{\Delta(G)}}{2}+\frac{3}{2}$, where $\Delta(G)$ is the maximum degree of nodes in $G$, i.e., $\Delta(G)=\max \{\operatorname{deg}(v) \mid v \in V(G)\}$. In the algorithm, an operator $>$ is defined among all nodes in $G$ as follows.

Definition 5.1: (Operator $>$ ): For any $u \in V(G)$ and $v \in$ $V(G), u>v$ iff either of the following two conditions holds. (1) $\operatorname{deg}(u, G)>\operatorname{deg}(v, G)$. (2) $\operatorname{deg}(u, G)=\operatorname{deg}(v, G)$ and $\operatorname{id}(u)>\operatorname{id}(v)$. The $>$ operator specifies a unique total order among all nodes in the graph $G$.

Using the $>$ operator, given a graph $G$, the algorithm in [7] scans all edges of $G$ on disk sequentially. For each edge $(u, v)$ scanned, if $u>v$, then $u$ is added to the vertex cover, otherwise, $v$ is added to the vertex cover.

In this paper, given $G_{i}\left(V_{i}, E_{i}\right)$, we adapt the external algorithm in [7] to construct $V_{i+1}$. We will further reduce the size of $V_{i+1}$ in Section VII. The basic algorithm to compute $V_{i+1}$ is shown in Algorithm 3. After initializing $V_{i+1}$ to be $\emptyset$ (line 1), two edge lists are created on disk, $E_{\text {in }}$ and $E_{\text {out }}$, by grouping incoming edges and out-going edges for each node in $G_{i}$ respectively using external sort (line 2-3). Since all edges are sorted in $E_{i n}$ and $E_{\text {out }}$, the degrees of all nodes in $G_{i}$ can be computed in $V_{d}$ by joining $E_{\text {in }}$ and $E_{\text {out }}$ using a single sequential scan of $E_{\text {in }}$ and $E_{\text {out }}$ (line 4). Next, we create another edge list $E_{d}$ with degree information of both nodes augmented on each edge, for the ease of comparison of nodes using the $>$ operator. $E_{d}$ can be created in three steps. Firstly, by joining $E_{\text {out }}$ and $V_{d}$ using a sequential scan, the degree of node $u$ can be augmented into each edge $(u, v)$ in $E_{d}$ 
(line 5). Secondly, $E_{d}$ is sorted by the non-augmented node of each edge (line 6). Thirdly, by joining $E_{d}$ and $V_{d}$ using a sequential scan, the degree of node $v$ can be augmented into each edge $(u, v)$ in $E_{d}$ (line 7$)$. After creating $E_{d}$, we only need to scan edges in $E_{d}$ sequentially once, and for each edge scanned, add the larger node compared by the $>$ operator into $V_{i+1}$ (line 8-9). Since $V_{i+1}$ may contain duplicate nodes, we sort $V_{i+1}$ by node ids and eliminate duplicate nodes by scanning $V_{i+1}$ once sequentially (line 10).

Lemma 5.2: The set $V_{i+1}$ computed in Algorithm 3 is recoverable and contractible.

Proof Sketch: Since Algorithm 3 computes a vertex cover $V_{i+1}$ of $G_{i}$, from Lemma 5.1, $V_{i+1}$ computed in Algorithm 3 is recoverable. Next, we prove that $V_{i+1}$ is contractible. We only need to prove that there exists a node $v$ such that $v \in V_{i}$ and $v \notin V_{i+1}$. By Definition 5.1, the operator $>$ specifies a unique total order among all nodes in $G_{i}$. Let $v$ be the smallest node in the total order defined by the operator $>, v$ cannot be added into $V_{i+1}$, because there does not exits an edge $(u, v)$ or an edge $(v, u)$ with $u>v$. Thus the lemma holds.

Theorem 5.1: The I/O complexity of Algorithm 3 is $O\left(\operatorname{sort}\left(\left|E_{i}\right|\right)+\operatorname{sort}\left(\left|V_{i}\right|\right)\right)$.

Proof Sketch: Omitted due to lack of space.

To construct $E_{i+1}$ : Given a graph $G_{i}\left(V_{i}, E_{i}\right)$ and the node set $V_{i+1}$ of the contracted graph $G_{i+1}$, we construct the edges $E_{i+1}$ of the contracted graph $G_{i+1}$. Since $V_{i+1}$ is constructed in a way such that the contractible and recoverable properties are satisfied, $E_{i+1}$ needs to be constructed that maintains the SCC-preservable property. In other words, after constructing $E_{i+1}$, for any two nodes $u \in V_{i+1}$ and $v \in V_{i+1}$, if $u$ and $v$ are in the same SCC in $G_{i}$, then $u$ and $v$ should be in the same SCC in $G_{i+1}$, and vice versa. Note that by removing a node $v$ from $G_{i}$, for any two nodes $u$ and $w$ in $V_{i+1}$, if $u$ can only reach $w$ through $v$ in $G_{i}$, then the connectivity of $u$ and $w$ is destroyed after the removal of node $v$. In order to maintain such connectivity in $G_{i+1}$, new edges need to be added after the removal of $v$. Suppose $u$ can reach $w$ through a path $\left(u \cdots v_{\text {in }}, v, v_{\text {out }}, \cdots w\right)$ in $G_{i}$, where $v_{\text {in }} \in \operatorname{nbr}_{i n}\left(v, G_{i}\right)$ and $v_{\text {out }} \in \operatorname{nbr}_{\text {out }}\left(v, G_{i}\right)$, after the removal of $v$, we can add a new edge $\left(v_{\text {in }}, v_{\text {out }}\right)$ in $E_{i+1}$, such that $u$ can still reach $w$ through a path $\left(u \cdots v_{i n}, v_{\text {out }}, \cdots w\right)$ in $G_{i+1}$. In order to do this, we need to make sure that both $v_{i n}$ and $v_{\text {out }}$ are in $G_{i+1}$. It is true because $V_{i+1}$ maintains the recoverable property such that for each removed node $v$, i.e., $v \in V_{i}-V_{i+1}$, all its neighbors in $G_{i}$ are in $V_{i+1}$, i.e., $\operatorname{nbr}\left(v, G_{i}\right) \subseteq V_{i+1}$.

An algorithm to construct $E_{i+1}$ can be designed as follows. For each node $v \in V_{i}-V_{i+1}$, and each pair of nodes $v_{\text {in }} \in \operatorname{nbr}_{i n}\left(v, G_{i}\right)$ and $v_{\text {out }} \in \mathrm{nbr}_{\text {out }}\left(v, G_{i}\right)$, remove the edge $\left(v_{i n}, v\right)$ and $\left(v, v_{\text {out }}\right)$, and add a new edge $\left(v_{i n}, v_{\text {out }}\right)$ in $E_{i+1}$. By doing this, for any pair of nodes $u \in V_{i+1}$ and $w \in V_{i+1}$, if $u$ can reach $w$ in $G_{i}, u$ can still reach $w$ in $G_{i+1}$. The edges are constructed to ensure that no connectivity of node pairs will be destroyed. We will prove it that the construction will introduce no new connectivity information among all nodes in

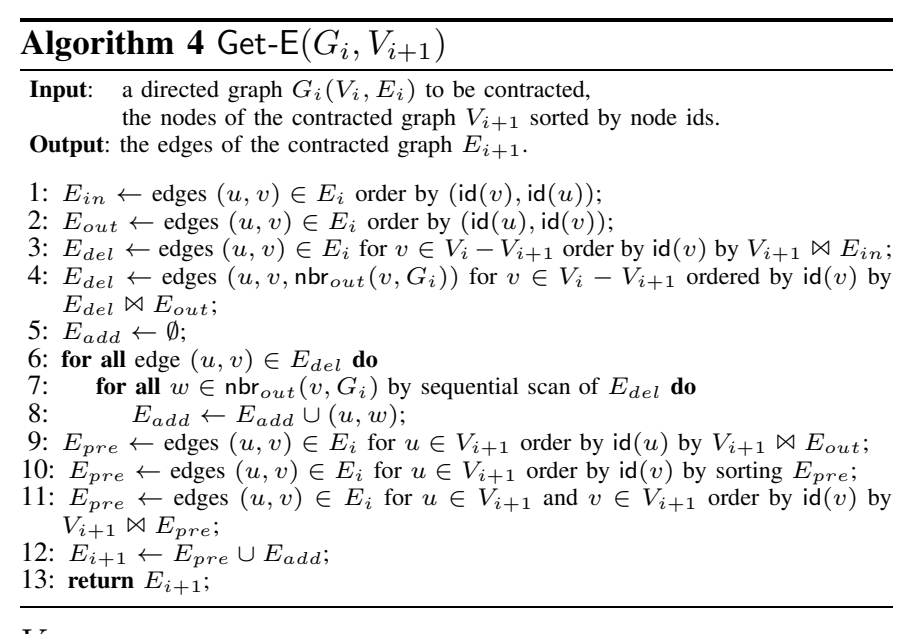
$V_{i+1}$.

Algorithm 4 shows how to construct $E_{i+1}$ externally, given $G_{i}\left(V_{i}, E_{i}\right)$ and $V_{i+1}$. As discussed above, $E_{i+1}$ consists of two parts, namely, the preserved edges in $G_{i}$ with both ends in $V_{i+1}$, denoted $E_{\text {pre }}$, and the newly added edges by removing nodes from $G_{i}$, denoted $E_{\text {add }}$. Let $E_{\text {in }}$ and $E_{\text {out }}$ be the edges of $G_{i}$ by grouping incoming and out-going edges for each node in $G_{i}$ respectively, which are the same as those used in Algorithm 3 (line 1-2), Algorithm 4 constructs $E_{a d d}$ in line 38 and constructs $E_{\text {pre }}$ in line $9-11$, and union $E_{a d d}$ and $E_{\text {pre }}$ to construct $E_{i+1}$ (line 12-13).

In order to construct $E_{a d d}$, the algorithm first identifies the set of incoming edges to be removed, denoted $E_{\text {del }}$, by joining $V_{i+1}$ and $E_{i n}$ using a single sequential scan of $V_{i+1}$ and $E_{i n}$ on disk (line 3 ). When scanning $V_{i+1}$ and $E_{i n}$, for each edge $(u, v) \in E_{i n}$, if $v \notin V_{i+1}$, then $(u, v)$ is added to $E_{d e l}$. After constructing the removed incoming edges, we augment the out-neighbors of $v$ into each incoming edge $(u, v) \in E_{\text {del }}$. This can be done using a single sequential scan of $E_{d e l}$ and $E_{\text {out }}$ (line 4 ). Line 5-8 construct $E_{a d d}$ using a single sequential scan of all edges in $E_{d e l}$. In $E_{d e l}$, for each node $v$ that is removed from $G_{i}$, each of its in-neighbors $u$ in $G_{i}$ is stored as an edge $(u, v)$, and its out-neighbors $\operatorname{nbr}_{\text {out }}\left(v, G_{i}\right)$ is also augmented in the edge $(u, v)$ with form $\left(u, v, \operatorname{nbr}_{\text {out }}\left(v, G_{i}\right)\right)$. When accessing each removed incoming edge $(u, v)$ of $v$ (line 6), the removed out-going edge $(v, w)$ of $v$ can be accessed in the same sequential scan of $E_{\text {del }}$ (line 7), and a new edge $(u, w)$ is added into $E_{a d d}$ (line 8).

The preserved edges $E_{\text {pre }}$ can be constructed in three steps. Firstly, by joining $V_{i+1}$ and $E_{\text {out }}$ using a sequential scan, all edges $(u, v)$ with $u \in V_{i+1}$ can be preserved in $E_{\text {pre }}$ (line 9). Secondly, we sort $E_{\text {pre }}$ such that all edges $(u, v) \in E_{\text {pre }}$ are sorted by id $(v)$ (line 10). Thirdly, by joining $V_{i+1}$ and $E_{\text {pre }}$ using a sequential scan, all edges $(u, v)$ with $u \in V_{i+1}$ and $v \in V_{i+1}$ can be preserved in $E_{\text {pre }}$ (line 11).

Lemma 5.3: The edge set $E_{i+1}$ constructed by Algorithm 4 is SCC-preservable.

Proof Sketch: We only need to prove for any nodes $u \in V_{i+1}$ and $w \in V_{i+1}, u \rightarrow w$ in $G_{i} \Leftrightarrow u \rightarrow w$ in $G_{i+1}$.

To prove $\Rightarrow$, for any path $p$ from $u$ to $w$ in $G_{i}$, we can find a path from $u$ to $w$ in $G_{i+1}$ as follows. For each node $v$ on 


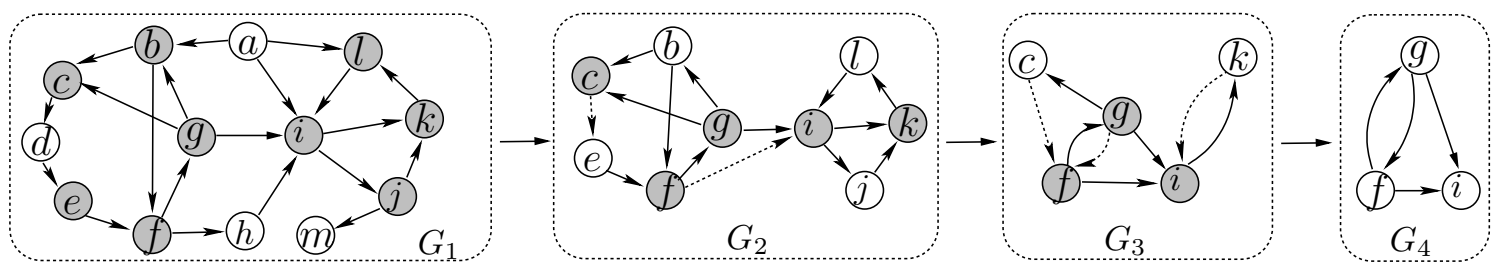

Fig. 4. Graph Contraction Example

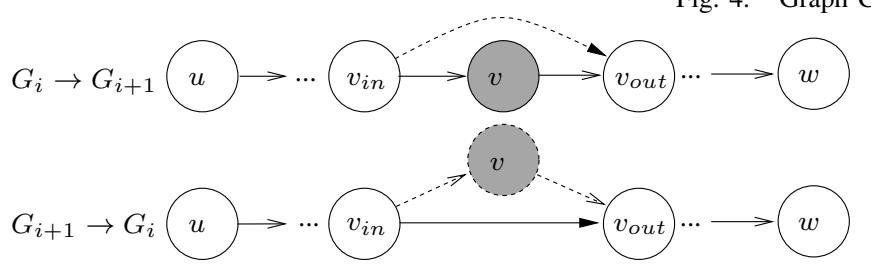

Fig. 3. Paths in $G_{i}$ and $G_{i+1}$

path $p$ with $v \notin V_{i+1}$, let $v_{\text {in }}$ and $v_{\text {out }}$ be the predecessor and successor of $v$ on path $p$, remove edge $\left(v_{i n}, v\right)$ and $\left(v, v_{\text {out }}\right)$ from path $p$ and add a new edge $\left(v_{\text {in }}, v_{\text {out }}\right)$ on $p$. From the construction of $E_{i+1}$, we have $\left(v_{i n}, v_{\text {out }}\right) \in E_{i+1}$. The new path $p$ is a path on $G_{i+1}$. The construction process is illustrated in the upper part of Fig. 3 .

To prove $\Leftarrow$, for any path $p$ from $u$ to $w$ in $G_{i+1}$, we can find a path from $u$ to $w$ in $G_{i}$ as follows. For each edge $\left(v_{\text {in }}, v_{\text {out }}\right)$ on path $p$, if $\left(v_{\text {in }}, v_{\text {out }}\right)$ is a newly added edge, from the construction of $E_{i+1}$, there exists a node $v \in V_{i}$ such that $\left(v_{\text {in }}, v\right) \in E_{i}$ and $\left(v, v_{\text {out }}\right) \in E_{i}$. We remove $\left(v_{\text {in }}, v_{\text {out }}\right)$ from $p$ and add two new edges $\left(v_{i n}, v\right)$ and $\left(v, v_{\text {out }}\right)$ on $p$. The new path $p$ is a path on $G_{i}$. The construction process is illustrated in the lower part of Fig. 3.

Theorem 5.2: The I/O complexity of Algorithm 4 is $O\left(\operatorname{sort}\left(\left|E_{i}\right|\right)+\operatorname{scan}\left(V_{i+1}\right)+\operatorname{scan}\left(\left|E_{i+1}\right|\right)\right)$.

Proof Sketch: Omitted due to lack of space.

Bounding Edge Size $\left|E_{i+1}\right|$ : From the above discussion, when a node $v$ is removed from $G_{i}$ when constructing $G_{i+1}, \operatorname{deg}_{i n}\left(v, G_{i}\right) \times \operatorname{deg}_{\text {out }}\left(v, G_{i}\right)$ new edges are added into $E_{i+1}$. When $\operatorname{deg}\left(v, G_{i}\right)$ is large, the number of newly added edges can be very large. However, from the construction of $V_{i+1}$, a node $v$ is removed only if for all $u \in \operatorname{nbr}\left(v, G_{i}\right)$, $\operatorname{deg}\left(u, G_{i}\right) \geq \operatorname{deg}\left(v, G_{i}\right)$. Thus, the degree of any removed node cannot be too large. The following two theorems give an upper bound of the degree for any removed node $v$, and an upper bound of the number of new edges in $E_{i+1}$ respectively.

Theorem 5.3: $\forall v \in V_{i}-V_{i+1}, \operatorname{deg}\left(v, G_{i}\right) \leq \sqrt{2 \times\left|E_{i}\right|}$.

Proof Sketch: For any $v \in V_{i}-V_{i+1}$ and $u \in \operatorname{nbr}\left(v, G_{i}\right)$, we have $\operatorname{deg}\left(u, G_{i}\right) \geq \operatorname{deg}\left(v, G_{i}\right)$. The total degrees of all nodes in $\operatorname{nbr}\left(v, G_{i}\right)$ is $\sum_{u \in \operatorname{nbr}\left(v, G_{i}\right)} \operatorname{deg}\left(u, G_{i}\right) \leq 2 \times$ $\left|E_{i}\right|$, since each edge in $E_{i}$ is counted at most twice in the summation. We also have $\sum_{u \in \operatorname{nbr}\left(v, G_{i}\right)} \operatorname{deg}\left(u, G_{i}\right) \geq$ $\sum_{u \in \operatorname{nbr}\left(v, G_{i}\right)} \operatorname{deg}\left(v, G_{i}\right)=\operatorname{deg}\left(v, G_{i}\right)^{2}$. Thus $\operatorname{deg}\left(v, G_{i}\right)^{2} \leq$ $2 \times\left|E_{i}\right|$. Theorem 5.3 holds.

Theorem 5.4: The number of new edges in $E_{i+1}$ is bounded by $\alpha_{i} \times\left|E_{i}\right|$, where $\alpha_{i}$ is the arboricity [11] of graph $G_{i}$.
Proof Sketch: By the construction of $E_{i+1}$, the number of new edges in $E_{i+1}$ is

$$
\begin{aligned}
& \Sigma_{v \in V_{i}-V_{i+1}} \operatorname{deg}_{i n}\left(v, G_{i}\right) \times \operatorname{deg}_{\text {out }}\left(v, G_{i}\right) \\
& \leq \Sigma_{v \in V_{i}-V_{i+1}} \operatorname{deg}_{i n}\left(v, G_{i}\right) \times \operatorname{deg}\left(v, G_{i}\right) \\
& =\Sigma_{v \in V_{i}-V_{i+1}} \Sigma_{u \in \operatorname{nbr}_{i n}\left(v, G_{i}\right)} \operatorname{deg}\left(v, G_{i}\right) \\
& =\Sigma_{v \in V_{i}-V_{i+1}} \Sigma_{u \in \operatorname{nbr} i n}\left(v, G_{i}\right) \min \left\{\operatorname{deg}\left(v, G_{i}\right), \operatorname{deg}\left(u, G_{i}\right)\right\} \\
& \leq \Sigma_{v \in V_{i}} \Sigma_{u \in \operatorname{nbr}}\left(v, G_{i}\right) \min \left\{\operatorname{deg}\left(v, G_{i}\right), \operatorname{deg}\left(u, G_{i}\right)\right\} \\
& =\Sigma_{(u, v) \in E_{i}} \min \left\{\operatorname{deg}\left(v, G_{i}\right), \operatorname{deg}\left(u, G_{i}\right)\right\} .
\end{aligned}
$$

According to [11], $\sum_{(u, v) \in E_{i}} \min \left\{\operatorname{deg}\left(v, G_{i}\right), \operatorname{deg}\left(u, G_{i}\right)\right\} \leq$ $\alpha_{i} \times\left|E_{i}\right|$ for any graph $G_{i}$.

As proved in [11], $\alpha_{i} \leq \min \left\{\left\lceil\sqrt{\left|E_{i}\right|} \mid, \operatorname{deg}_{\max }\right\}\right.$ for any graph $G_{i}$, where $\operatorname{deg}_{\max }$ is the maximum degree for all nodes in $G_{i}$. In practice, $\alpha_{i}$ is usually small. For example, $\alpha_{i}=O(1)$ if $G_{i}$ is a planar graph. Note that in Theorem 5.4, the upper bound $\alpha_{i} \times\left|E_{i}\right|$ is very loose, since it amplifies $\Sigma_{v \in V_{i}-V_{i+1}} \Sigma_{u \in \operatorname{nbr}_{i n}\left(v, G_{i}\right)} \min \left\{\operatorname{deg}\left(v, G_{i}\right), \operatorname{deg}\left(u, G_{i}\right)\right\}$ to $\Sigma_{v \in V_{i}} \Sigma_{u \in \operatorname{nbr}_{i n}\left(v, G_{i}\right)} \min \left\{\operatorname{deg}\left(v, G_{i}\right), \operatorname{deg}\left(u, G_{i}\right)\right\}$ in the second $\leq$ of Eq. (1), and as analyzed in Theorem 5.3, the set $V_{i}-V_{i+1}$ only contains nodes with small degrees in $G_{i}$. In section VII, we propose techniques to further reduce $\left|E_{i+1}\right|$, and in our experiments, it is even possible that $\left|E_{i+1}\right|<\left|E_{i}\right|$ for a certain graph $G_{i}$.

Example 5.1: Fig. 4 shows the graph contraction phase for $G$ in Fig. 1. The grey nodes in each $G_{i}$ are the set of nodes preserved in $G_{i+1}$, and the dashed edges in each $G_{i}$ are the newly added edges when constructing $G_{i}$ from $G_{i-1}$. In $G_{1}$ (= $G)$, node $b$ is preserved in $V_{2}$, since there is an edge $(a, b)$ with $\operatorname{deg}\left(b, G_{1}\right)>\operatorname{deg}\left(a, G_{1}\right)$, thus $b>a$ by operator $>$ defined in Definition 5.1. $d \in V_{1}-V_{2}$ because for its two neighbors $c$ and $e, c>d$ and $e>d$. After removing $d$, a new edge $(c, e)$ is added in $G_{2}$ since $c \in \operatorname{nbr}_{i n}\left(d, G_{1}\right)$ and $e \in \operatorname{nbr}_{\text {out }}\left(d, G_{1}\right)$. Using such a way, the newly constructed $G_{2}$ has 9 nodes and 14 edges by removing parallel edges and self circles. In a similar way, $G_{3}$ can be constructed from $G_{2}$ with $\left|V_{3}\right|=5$ and $\left|E_{3}\right|=8 . G_{4}$ can be constructed from $G_{3}$ with $\left|V_{4}\right|=3$ and $\left|E_{4}\right|=4$. Suppose the main memory can only keep three nodes. The graph contraction phase stops after $G_{4}$ is constructed, since $G_{4}$ can be processed using Semi-SCC.

\section{GRAPH EXPANSION}

The graph contraction phase stops when all nodes of the contracted graph $G_{i+1}$ can fit in memory, such that the SCCs of $G_{i+1}$ can be computed using the semi-external algorithm Semi-SCC. The graph expansion phase computes all SCCs of $G_{i}$ using the information computed in $G_{i+1}$ with decreasing $i$ iteratively. Given graph $G_{i}\left(V_{i}, E_{i}\right)$ and $G_{i+1}\left(V_{i+1}, E_{i+1}\right)$, 
suppose all SCCs of nodes in $V_{i+1}$ are computed, denoted as $\mathrm{SCC}_{i+1}$, we discuss computing $\mathrm{SCC}_{i}$, the set of all SCC $s$ of nodes in $V_{i}$. According to the SCC-preservable property of $G_{i+1}$ in Lemma 5.3, we only need to compute $\operatorname{SCC}\left(v, G_{i}\right)$ for each $v \in V_{i}-V_{i+1}$, since $\operatorname{SCC}\left(v, G_{i}\right)=\operatorname{SCC}\left(v, G_{i+1}\right)$ for each $v \in V_{i+1}$ according to Lemma 5.3. We start with the following lemma.

Lemma 6.1: Given any two nodes $u \in V_{i+1}$ and $w \in V_{i+1}$ (possibly $u=w$ ) with $\operatorname{SCC}\left(u, G_{i}\right)=\operatorname{SCC}\left(w, G_{i}\right)$ in graph $G_{i}$, for any node $v \in V_{i}-V_{i+1}, \operatorname{SCC}\left(u, G_{i}\right)=\operatorname{SCC}\left(w, G_{i}\right)=$ $\operatorname{SCC}\left(v, G_{i}\right) \Leftrightarrow u \rightarrow v$ and $v \rightarrow w$ in $G_{i}$.

Proof Sketch: $\Rightarrow$ is trivial. We prove $\Leftarrow$. Because $\operatorname{SCC}\left(u, G_{i}\right)=\operatorname{SCC}\left(w, G_{i}\right)$, we have $u \leftrightarrow w$ in $G_{i}$. Since $u \rightarrow v$ and $v \rightarrow w$ in $G_{i}$, we can derive that $v \rightarrow w \rightarrow u \rightarrow v$ in $G_{i}$. Thus $\operatorname{SCC}\left(u, G_{i}\right)=\operatorname{SCC}\left(w, G_{i}\right)=\operatorname{SCC}\left(v, G_{i}\right)$.

Lemma 6.1 suggests a way to compute $\operatorname{SCC}\left(v, G_{i}\right)$ for a node $v \in V_{i}-V_{i+1}$ : to find two nodes $u$ and $w$ (possibly the same) in $V_{i+1}$ with $\operatorname{SCC}\left(u, G_{i}\right)=\operatorname{SCC}\left(w, G_{i}\right)$ and $u \rightarrow v$ and $v \rightarrow w$ in $G_{i}$. However, $u$ and $w$ are not easy to find, and $u \rightarrow v$ and $v \rightarrow w$ are not easy to compute. In the following, we show that it is enough to find $u$ from $\operatorname{nbr}_{i n}\left(v, G_{i}\right)$ and find $w$ from $\operatorname{nbr}_{\text {out }}\left(v, G_{i}\right)$. We first investigate some properties of SCC $s$ in $\operatorname{nbr}_{i n}\left(v, G_{i}\right)$ and $\operatorname{nbr}_{\text {out }}\left(v, G_{i}\right)$ in Lemma 6.2 and Lemma 6.3.

Lemma 6.2: For any node $v \in V_{i}-V_{i+1}$, if $\operatorname{SCC}\left(\mathrm{nbr}_{i n}\left(v, G_{i}\right)\right.$, $\left.G_{i}\right) \cap \operatorname{SCC}\left(\operatorname{nbr}_{\text {out }}\left(v, G_{i}\right), G_{i}\right) \neq \emptyset$, then $\operatorname{SCC}\left(\operatorname{nbr}_{i n}\left(v, G_{i}\right)\right.$, $\left.G_{i}\right) \cap \operatorname{SCC}\left(\operatorname{nbr}_{\text {out }}\left(v, G_{i}\right), G_{i}\right)=\left\{\operatorname{SCC}\left(v, G_{i}\right)\right\}$.

Proof Sketch: Suppose there exists $v_{i n} \in \operatorname{nbr}_{i n}\left(v, G_{i}\right)$ and $v_{\text {out }} \in \operatorname{nbr}_{\text {out }}\left(v, G_{i}\right)$ with $\operatorname{SCC}\left(v_{\text {in }}, G_{i}\right)=\operatorname{SCC}\left(v_{\text {out }}, G_{i}\right)$, since $v_{\text {in }} \rightarrow v$ and $v \rightarrow v_{\text {out }}$, from Lemma 6.1, we have $\operatorname{SCC}\left(v_{i n}, G_{i}\right)=\operatorname{SCC}\left(v_{\text {out }}, G_{i}\right)=\operatorname{SCC}\left(v, G_{i}\right)$ in graph $G_{i}$. As a result, $\operatorname{SCC}\left(\operatorname{nbr}_{i n}\left(v, G_{i}\right), G_{i}\right) \cap \operatorname{SCC}\left(\operatorname{nbr}_{\text {out }}\left(v, G_{i}\right), G_{i}\right)$ $=\left\{\operatorname{SCC}\left(v, G_{i}\right)\right\}$

Lemma 6.3: For any node $v \in V_{i}-V_{i+1}$, if there exists another node $u \in V_{i}$ with $\operatorname{SCC}\left(v, G_{i}\right)=\operatorname{SCC}\left(u, G_{i}\right)$, then $\operatorname{SCC}\left(v, G_{i}\right) \in \operatorname{SCC}\left(\operatorname{nbr}_{i n}\left(v, G_{i}\right), G_{i}\right)$ and $\operatorname{SCC}\left(v, G_{i}\right) \in$ $\operatorname{SCC}\left(\operatorname{nbr}_{\text {out }}\left(v, G_{i}\right), G_{i}\right)$.

Proof Sketch: Since $u \neq v$ and $\operatorname{SCC}\left(v, G_{i}\right)=\operatorname{SCC}\left(u, G_{i}\right)$, there exists $v_{\text {in }}$ and $v_{\text {out }}$, such that $u \rightarrow v$ through a path $\left(u, \cdots, v_{i n}, v\right)$ with $v_{i n} \in \operatorname{nbr}_{i n}\left(v, G_{i}\right)$, and $v \rightarrow u$ through a path $\left(v, v_{\text {out }}, \cdots, u\right)$ with $v_{\text {out }} \in \operatorname{nbr}_{\text {out }}\left(v, G_{i}\right)$. Thus $\operatorname{SCC}\left(v, G_{i}\right)=\operatorname{SCC}\left(v_{i n}, G_{i}\right)=\operatorname{SCC}\left(v_{\text {out }}, G_{i}\right)$. As a result, $\operatorname{SCC}\left(v, G_{i}\right) \in \operatorname{SCC}\left(\operatorname{nbr}_{i n}\left(v, G_{i}\right), G_{i}\right)$ and $\operatorname{SCC}\left(v, G_{i}\right) \in$ $\operatorname{SCC}\left(\operatorname{nbr}_{\text {out }}\left(v, G_{i}\right), G_{i}\right)$.

According to the recoverable property of $G_{i+1}$ in Lemma 5.2, for any node $v \in V_{i}-V_{i+1}, \operatorname{nbr}_{i n}\left(v, G_{i}\right) \subseteq V_{i+1}$ and $\operatorname{nbr}_{\text {out }}\left(v, G_{i}\right) \subseteq V_{i+1}$. As a result, both $\operatorname{SCC}\left(\mathrm{nbr}_{i n}\left(v, G_{i}\right), G_{i}\right)$ and $\operatorname{SCC}\left(\operatorname{nbr}_{\text {out }}\left(v, G_{i}\right), G_{i}\right)$ are computed in $G_{i+1}$. The following lemma shows that $\operatorname{SCC}(v)$ can be computed using $\operatorname{SCC}\left(\mathrm{nbr}_{i n}\left(v, G_{i}\right), G_{i}\right)$ and $\operatorname{SCC}\left(\operatorname{nbr}_{\text {out }}\left(v, G_{i}\right), G_{i}\right)$ only.

Lemma 6.4: For any node $v \in V_{i}-V_{i+1}, \operatorname{SCC}\left(v, G_{i}\right)$ can be computed using $\operatorname{nbr}_{i n}\left(v, G_{i}\right)$ and $\operatorname{nbr}_{\text {out }}\left(v, G_{i}\right)$ only.

Proof Sketch: There are two situations: 1) If $\operatorname{SCC}\left(\mathrm{nbr}_{i n}(v\right.$,

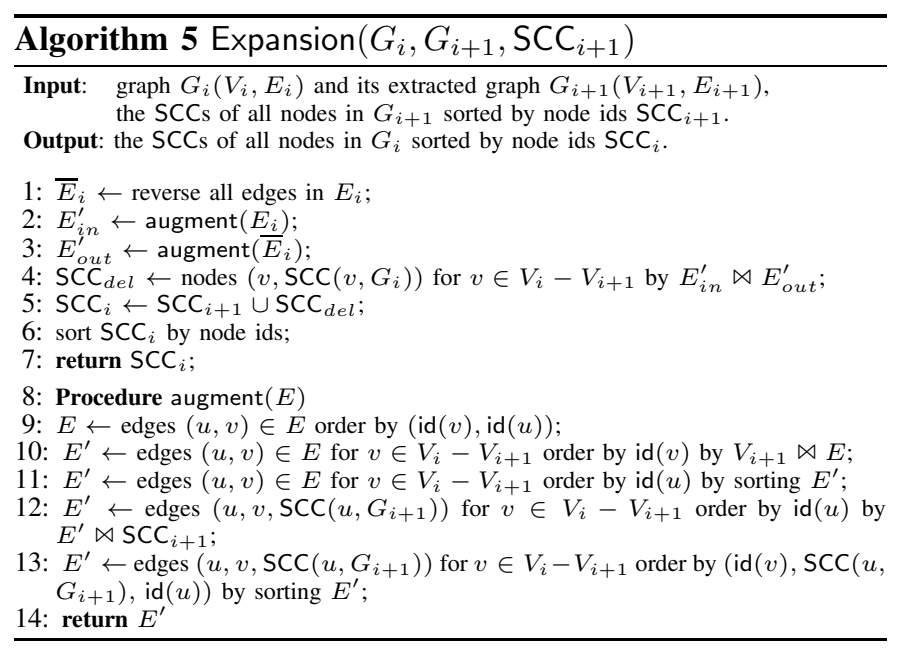

$\left.\left.G_{i}\right), G_{i}\right) \cap \operatorname{SCC}\left(\operatorname{nbr}_{\text {out }}\left(v, G_{i}\right), G_{i}\right) \neq \emptyset$, according to Lemma 6.2, $\operatorname{SCC}\left(v, G_{i}\right)$ can be calculated using $\operatorname{SCC}\left(\mathrm{nbr}_{i n}\left(v, G_{i}\right)\right.$, $\left.G_{i}\right) \cap \operatorname{SCC}\left(\operatorname{nbr}_{\text {out }}\left(v, G_{i}\right), G_{i}\right)$. 2) If SCC $\left(\operatorname{nbr}_{i n}\left(v, G_{i}\right), G_{i}\right) \cap$ $\operatorname{SCC}\left(\operatorname{nbr}_{\text {out }}\left(v, G_{i}\right), G_{i}\right)=\emptyset$, according to Lemma 6.3, there does not exist another node $u \in G_{i}$ with $\operatorname{SCC}\left(v, G_{i}\right)=\operatorname{SCC}($ $\left.u, G_{i}\right)$. As a result, $v$ is an SCC with a single node in $G_{i}$. $\square$

Given $G_{i}\left(V_{i}, E_{i}\right), G_{i+1}\left(V_{i+1}, E_{i+1}\right)$, and $\mathrm{SCC}_{i+1}$, our external algorithm to compute $\mathrm{SCC}_{i}$ is shown in Algorithm 5. In order to join $\operatorname{nbr}_{i n}\left(v, G_{i}\right)$ and $\operatorname{nbr}_{\text {out }}\left(v, G_{i}\right)$ for each removed node $v$, the algorithm augments the SCC information into the in-neighbors of the removed nodes using augment $\left(E_{i}\right)$ (line 2), and augments the SCC information into the outneighbors of the removed nodes using augment $\left(\bar{E}_{i}\right)$ (line 3 ), where $\bar{E}_{i}$ is generated by reversing every edge in $E_{i}$. Note that in-neighbors in $\bar{E}_{i}$ become out-neighbors in $E_{i}$. The procedure to augment the SCC into the in-neighbors of $E_{i} / \bar{E}_{i}$ of each removed node is shown in line 8-14. In the procedure, a new edge set $E^{\prime}$ that keeps the incoming edges of only the removed nodes $V_{i}-V_{i+1}$ is created by joining $E$ and $V_{i+1}$ (line 10). In line 11 , edges $(u, v)$ in $E^{\prime}$ are sorted by $\operatorname{id}(u)$ in order to augment $\operatorname{SCC}(u)$. In line $12, \operatorname{SCC}(u)$ is augmented in each edge $(u, v)$ in $E^{\prime}$ by sequential scan of $E^{\prime}$ and $\mathrm{SCC}_{i+1}$. In line 13 , edges $(u, v, \operatorname{SCC}(u))$ in $E^{\prime}$ are sorted by $(\operatorname{id}(v), \operatorname{SCC}(u), \operatorname{id}(u))$ to put the in-neighbors of each node in $V_{i}-V_{i+1}$ together in order to compute $\operatorname{nbr}_{i n}\left(v, G_{i}\right) \cap \operatorname{nbr}_{\text {out }}\left(v, G_{i}\right)$ efficiently. After augmenting the SCCs into the in-neighbors and out-neighbors of the removed nodes, in line 4, the SCC $s$ of all nodes $v \in V_{i}-V_{i+1}$ can be computed using $\operatorname{nbr}_{i n}\left(v, G_{i}\right) \cap \operatorname{nbr}_{\text {out }}\left(v, G_{i}\right)$ by a sequential scan of $E_{\text {in }}^{\prime}$ and $E_{\text {out }}^{\prime}$. In line 5, by combining SCCs in $V_{i}-V_{i+1}$ and SCCs in $V_{i+1}$ computed in $G_{i+1}$, the SCCs for all nodes in $G_{i}$ can be computed as $\mathrm{SCC}_{i}$. Finally, all nodes in $\mathrm{SCC}_{i}$ are sorted (line 6) and $\mathrm{SCC}_{i}$ is returned (line 7).

Theorem 6.1: The I/O complexity of Algorithm 5 is $O\left(\operatorname{scan}\left(\left|V_{i+1}\right|\right)+\operatorname{sort}\left(\left|E_{i}\right|\right)+\operatorname{sort}\left(\left|V_{i}\right|\right)\right)$.

Proof Sketch: Omitted due to lack of space.

Theorem 6.2: Algorithm 5 computes all SCCs of $G_{i}$.

Proof Sketch: For node $v \in V_{i+1}, \operatorname{SCC}\left(v, G_{i}\right)=$ $\operatorname{SCC}\left(v, G_{i+1}\right)$ according to the SCC-preservable property in Lemma 5.3. For node $v \in V_{i}-V_{i+1}, \operatorname{SCC}\left(v, G_{i}\right)$ is correctly 


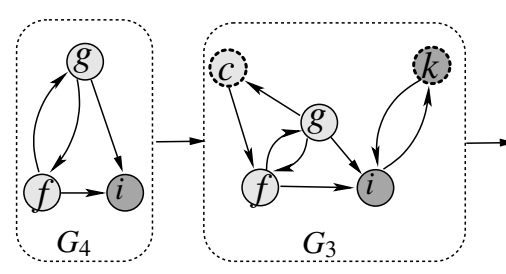

computed according to Lemma 6.4.

Example 6.1: Fig. 5 shows the process of the graph expansion phase, to expand the graphs in order of $G_{4}, G_{3}, G_{2}$, and $G_{1}$ generated in the graph contraction phase in Fig. 4. The dashed circles in each graph $G_{i}$ are the removed nodes when constructing $G_{i+1}$ from $G_{i}$. By applying the Semi-SCC algorithm on $G_{4}$, two SCCs are computed, namely, $S C C_{1}$ denoted by light gray nodes, and $S C C_{2}$ denoted by dark gray nodes. In $G_{3}$, for node $c \in V_{3}-V_{4}$, we have $g \in \operatorname{nbr}_{i n}\left(c, G_{3}\right)$ and $f \in \operatorname{nbr}_{\text {out }}\left(c, G_{3}\right)$ with $\operatorname{SCC}\left(g, G_{3}\right)=\operatorname{SCC}\left(f, G_{3}\right)=S C C_{1}$. Thus $\operatorname{SCC}\left(c, G_{3}\right)=S C C_{1}$. Similarly, the SCCs of node $k$ in $G_{3}$, and nodes $b, e, l, j$ in $G_{2}$ can be computed. In $G_{1}$, for node $h, \operatorname{SCC}\left(\operatorname{nbr}_{i n}\left(h, G_{1}\right), G_{1}\right)=\left\{S C C_{1}\right\}$ and $\operatorname{SCC}\left(\operatorname{nbr}_{\text {out }}\left(h, G_{1}\right), G_{1}\right)=\left\{S C C_{2}\right\}, \operatorname{SCC}\left(\operatorname{nbr}_{\text {in }}\left(h, G_{1}\right), G_{1}\right)$ $\cap \operatorname{SCC}\left(\operatorname{nbr}_{\text {out }}\left(h, G_{1}\right), G_{1}\right)=\emptyset$. Thus node $h$ in $G_{1}$ is an SCC with a single node. Finally, there are two SCC $s S C C_{1}$ and $S C C_{2}$ with 6 and 4 nodes respectively.

\section{I/O Cost Minimization}

In this section, we show how to optimize our contractionexpansion based Ext-SCC approach by further reducing the I/O cost. The I/O cost can be reduced in two ways: (1) to reduce the number of graphs constructed in the graph contraction phase, and (2) to reduce the number of nodes and edges for each graph $G_{i}$ constructed in the $i$-th iteration. According to the stop condition of graph contraction, reducing the number of graphs is equivalent to reducing the number of nodes $\left|V_{i}\right|$ in each graph $G_{i}$. Thus, the key point to reduce the I/O cost of the Ext-SCC algorithm is to reduce the number of nodes $\left|V_{i}\right|$ and number of edges $\left|E_{i}\right|$ in each graph $G_{i}$. In the following, we introduce techniques to reduce the nodes and edges when constructing $G_{i+1}$ from $G_{i}$.

Node Reduction: Given graph $G_{i}$, when constructing $G_{i+1}$, suppose $V_{i+1}$ has already been computed using Algorithm 3, the following two types of nodes can be removed from $V_{i+1}$.

- (Type-1): For a node $v \in V_{i+1}$, if there does not exit another node $u \in V_{i} \operatorname{such}$ that $\operatorname{SCC}\left(v, G_{i}\right)=\operatorname{SCC}\left(u, G_{i}\right)$, $v$ can be removed from $V_{i+1}$.

- (Type-2): For a node $v \in V_{i+1}$, if $\operatorname{nbr}\left(v, G_{i}\right) \subseteq V_{i+1}, v$ can be removed from $V_{i+1}$.

A Type- 1 node $v$ can be removed because $v$ itself is an SCC with a single node in $G_{i}$, and there is no need to include $v$ in later iterations as $v$ cannot be combined with other nodes to form new SCCs in later iterations. A Type-2 node $v$ can be removed because when $\operatorname{nbr}\left(v, G_{i}\right) \subseteq V_{i+1}, V_{i+1}-\{v\}$ is still a vertex cover of $G_{i}$. According to the node selection condition in Lemma 5.1, $V_{i+1}-\{v\}$ is a valid node set of $G_{i+1}$. Type- 2 nodes are order sensitive, i.e., if node $u$ and $v$

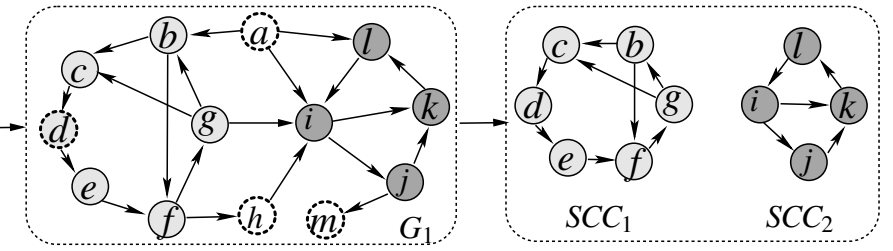

are Type- 2 nodes, after $u$ is removed, $v$ may not be a Type- 2 node. Note that our aim is to reduce nodes in $V_{i+1}$ without introducing new I/O cost, thus we only reduce those Type-1 and Type- 2 nodes that are easy to be identified. The following lemma can be used to reduce Type-1 nodes.

Lemma 7.1: Any node $v$ with $\operatorname{deg}_{\text {in }}\left(v, G_{i}\right)=0$ or $\operatorname{deg}_{\text {out }}\left(v, G_{i}\right)=0$ is a Type-1 node.

Proof Sketch: Omitted due to lack of space.

To remove Type- 1 node $v$ from $V_{i+1}$ with $\operatorname{deg}_{i n}\left(v, G_{i}\right)=0$ or $\operatorname{deg}_{\text {out }}\left(v, G_{i}\right)=0$, when generating $V_{d}$ in line 4 of Algorithm 3, by joining $E_{\text {in }}$ and $E_{\text {out }}$, we only keep the nodes with both $\operatorname{deg}_{i n}\left(v, G_{i}\right)>0$ and $\operatorname{deg}_{\text {out }}\left(v, G_{i}\right)>0$ in $V_{d}$. Since $V_{i+1}$ is generated from $E_{d}$ which is computed using $V_{d}$, all nodes $v$ with $\operatorname{deg}_{i n}\left(v, G_{i}\right)=0$ or $\operatorname{deg}_{\text {out }}\left(v, G_{i}\right)=0$ will be removed in $V_{i+1}$ in Algorithm 3. Such an operation does not generate any extra I/O cost in Algorithm 3.

In order to reduce Type- 2 nodes, when scanning all edges in $E_{d}$ in line 8-9 of Algorithm 3, for each edge $(u, v)$ scanned, suppose $v>u$, before adding $v$ into $V_{i+1}$, we check whether $u$ has been added into $V_{i+1}$. If so, edge $(u, v)$ has been covered by node $u$ and there is no need to add $v$ into $V_{i+1}$ to cover the edge $(u, v)$ again. The situation for $u>v$ can be handled similarly. In such a way, Type- 2 nodes can be effectively reduced in $V_{i+1}$. However, such a solution needs to check whether $u \in V_{i+1}$ using a dictionary $T$ which many not reside entirely in the main memory. Suppose $T$ can only hold $s$ nodes in memory, since a node with higher degree are less possible to be removed from $V_{i+1}$, when adding nodes into $T$, we only maintain the top $s$ smallest nodes using operator $>$ in $T$, to make sure that $T$ can reside entirely in the main memory. By doing so, we can reduce the number of Type- 2 nodes in $V_{i+1}$ without generating any extra $\mathrm{I} / \mathrm{O}$ cost in Algorithm 3.

Edge Reduction: Given $G_{i}\left(V_{i}, E_{i}\right)$, we introduce two ways to reduce the number of edges when generating $G_{i+1}$ in the $i$-th graph contraction phase without increasing the I/O complexity. Although $\left|V_{i}\right|<\left|V_{i-1}\right|$, it is possible that $\left|E_{i}\right|>\left|E_{i-1}\right|$. We develop two methods to reduce the edge size in order to reduce the intermediate results. We will discuss the efficiency in our performance studies.

Firstly, for parallel edges with the same form $(u, v)$, only one of them needs to be kept in $E_{i+1}$. Such edges can be reduced in a lazy way, when generating $E_{i n}$ in the next iteration of Get-E (line 2 of Algorithm 3). A sequential scan of $E_{\text {in }}$ needs to be added after line 2 of Algorithm 3 to eliminate parallel edges in $E_{i n}$. In addition, it is straightforward that each edge $(u, w)$ with $u=w$ can be removed from $E_{i+1}$. This can be done in line 8 of Algorithm 4, by checking whether 


\begin{tabular}{|l|l|l|}
\hline Parameter & Range & Default \\
\hline Size of $|V|$ & $25 \mathrm{M}, 50 \mathrm{M}, 100 \mathrm{M}, 150 \mathrm{M}, 200 \mathrm{M}$ & $100 \mathrm{M}$ \\
\hline Average Degree $D$ & $2,3,4,5,6$ & 4 \\
\hline Memory Size $M$ & $200 \mathrm{M}, 300 \mathrm{M}, 400 \mathrm{M}, 500 \mathrm{M}, 600 \mathrm{M}$ & $400 \mathrm{M}$ \\
\hline Size of Massive-SCC & $200 \mathrm{~K}, 300 \mathrm{~K}, 400 \mathrm{~K}, 500 \mathrm{~K}, 600 \mathrm{~K}$ & $400 \mathrm{~K}$ \\
\hline Size of Large-SCC & $4 \mathrm{~K}, 6 \mathrm{~K}, 8 \mathrm{~K}, 10 \mathrm{~K}, 12 \mathrm{~K}$ & $8 \mathrm{~K}$ \\
\hline Size of Small-SCC & $20,30,40,50,60$ & 40 \\
\hline Number of Massive-SCC $s$ & 1 & 1 \\
\hline Number of Large-SCC $s$ & $30,40,50,60,70$ & 50 \\
\hline Number of Small-SCC $s$ & $6 \mathrm{~K}, 8 \mathrm{~K}, 10 \mathrm{~K}, 12 \mathrm{~K}, 14 \mathrm{~K}$ & $10 \mathrm{~K}$ \\
\hline
\end{tabular}

TABLE I

RANGE AND DEFAULT VALUE FOR PARAMETERS

$u=w$ before adding $(u, w)$ to $E_{a d d}$.

Secondly, using operator $>$, according to Theorem 5.3, nodes with small degrees are removed when constructing $V_{i+1}$, and for each removed node $v \in V_{i}-V_{i+1}$, $\operatorname{deg}_{i n}\left(v, G_{i}\right) \times$ $\operatorname{deg}_{\text {out }}\left(v, G_{i}\right)$ new edges are added into $E_{i+1}$. By considering $\operatorname{deg}_{\text {in }}\left(v, G_{i}\right) \times \operatorname{deg}_{\text {out }}\left(v, G_{i}\right)$ in the operator $>,\left|E_{i+1}\right|$ can be further reduced. We redefine the operator $>$ as follows.

Definition 7.1: (Operator $>$ ): For any $u \in V(G)$ and $v \in$ $V(G), u>v$ iff one of the following three conditions holds. (1) $\operatorname{deg}(u, G)>\operatorname{deg}(v, G)$. (2) $\operatorname{deg}(u, G)=\operatorname{deg}(v, G)$ and $\operatorname{deg}_{\text {in }}(u, G) \times \operatorname{deg}_{\text {out }}(u, G)>\operatorname{deg}_{\text {in }}(v, G) \times \operatorname{deg}_{\text {out }}(v, G)$. (3) $\operatorname{deg}(u, G)=\operatorname{deg}(v, G)$ and $\operatorname{deg}_{\text {in }}(u, G) \times \operatorname{deg}_{\text {out }}(u, G)=$ $\operatorname{deg}_{\text {in }}(v, G) \times \operatorname{deg}_{\text {out }}(v, G)$ and $\operatorname{id}(u)>\operatorname{id}(v)$. The $>$ operator specifies a unique total order among all nodes in the graph $G$.

In Algorithm 3, in order to make use of the new $>$ operator in line 9 , when generating $V_{d}$ in line 4 , both $\operatorname{deg}\left(v, G_{i}\right)$ and $\operatorname{deg}_{i n}\left(v, G_{i}\right) \times \operatorname{deg}_{\text {out }}\left(v, G_{i}\right)$ need to be computed in $V_{d}$ in line 4, and augmented in all nodes in $E_{d}$ in line 5-7.

\section{Performance Studies}

In this section, we conduct experimental studies by comparing four external algorithms for SCC computation, namely, the external contraction based EM-SCC [13], the external DFS based DFS-SCC [8], our external contraction-expansion based algorithm Ext-SCC (Algorithm 2), and our algorithm ExtSCC-Op by applying the optimization techniques introduced in Section VII in Ext-SCC. All the algorithms are implemented using Visual C++ 2005 and tested on a PC with Intel Core2 Quar 2.66GHz CPU and 3.5GB memory running Windows $\mathrm{XP}$. The disk block size is $256 \mathrm{~KB}$. The default memory size is $400 M$. For the semi-external algorithm Semi-SCC used in Ext-SCC, we apply the algorithm 1PB-SCC introduced in [26], which is currently the most I/O efficient semi-external algorithm for SCC computation. The 1PB-SCC algorithm needs to hold $2 \times|V(G)|$ plus one disk block in the main memory, that is $M=4 \times(2 \times|V(G)|)+256 K$ where 4 is the number of bytes to keep a node in memory. We set the max time cost to be 24 hours. If a test does not stop in the time limit, we will denote it using INF. In our experiments, we do not show the results of EM-SCC since it cannot stop in all cases.

Datasets: In our experiments, we use a real large web graph and several synthetic datasets. The real web graph is WEBSPAM-UK2007 ${ }^{4}$, which consists of $105,896,555$ web-

\footnotetext{
${ }^{4}$ barcelona.research.yahoo.net/webspam/datasets/uk2007/links/
}

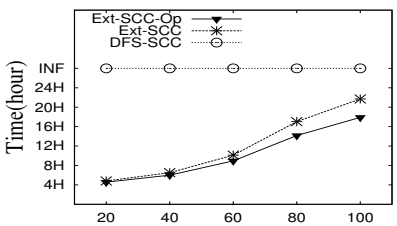

(a) Time (Vary Graph Size)

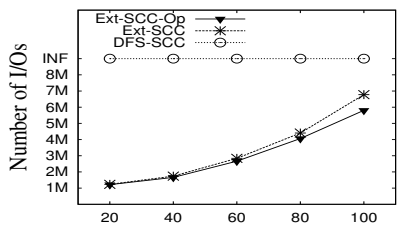

(b) I/Os (Vary Graph Size)
Fig. 6. WEBSPAM-UK2007: Varying Graph Size (Percent)

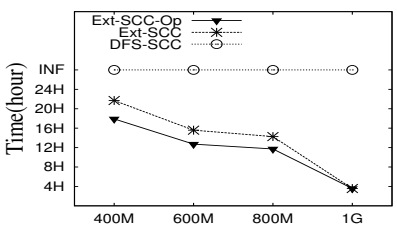

(a) Time (Vary Memory)

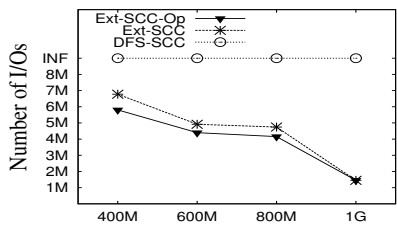

(b) I/Os (Vary Memory)
Fig. 7. WEBSPAM-UK2007: Varying Memory Size

pages in 114,529 hosts in the .UK domain. The graph contains $105,895,908$ nodes and 3,738,733,568 edges, with the average degree 35 per node. For synthetic data, we generate 3 different kinds of datasets, denoted Massive-SCC, Large-SCC, and Small-SCC, containing different sizes of SCCs. The graphs contain nodes from $25 \mathrm{M}$ to $200 \mathrm{M}$ with average degree varying from 2 to 6 . A synthetic graph is generated as follows. We construct a graph $G$ by randomly selecting all nodes in SCCs first. Then we add edges among the nodes in an SCC until all nodes form an SCC. Finally, additional random nodes and edges are added to the graph. The parameters for synthetic datasets and their default values are shown in Table I.

Exp-1 (Performance on WEBSPAM-UK2007): Fig. 6(a) and Fig. 6(b) show the time and I/O costs when varying the number of edges of WEBSPAM-UK2007 from $20 \%$ to $100 \%$ respectively. DFS-SCC cannot stop in the time limit even if the graph contains only $20 \%$ of the edges. When $|E|$ increases, the time and I/O consumptions for both Ext-SCC and Ext-SCC-Op increase. The reasons are twofold. Firstly, when $|E|$ increases, the number of iterations in graph contraction increases. This is because when number of edges $|E|$ increases, according to the node selection scheme to construct $V_{i+1}$ in Algorithm 3, more nodes will be selected in $V_{i+1}$, thus more iterations are needed according to the stop condition of graph contraction in Ext-SCC. Secondly, when $|E|$ increases, the cost to sort and scan edges in each iteration increases, thus more time and I/Os are consumed in each iteration. Ext-SCC-Op outperforms Ext-SCC in all cases since more nodes/edges are removed in each iteration in Ext-SCC-Op.

We vary the memory size from $400 M$ to $1 G$. The results are shown in Fig. 7(a) and Fig. 7(b) for time and I/O costs respectively. When the memory size increases, the time and I/O costs for both Ext-SCC and Ext-SCC-Op decrease. There are two reasons. Firstly, when the memory size increases, the stop condition for graph contraction is easier to be satisfied since more nodes can fit in memory. Secondly, when the memory size increases, the costs of the external sorts in both graph contraction and graph expansion phases decrease. Ext-SCC-Op 


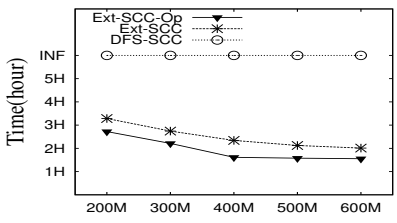

(a) Time (Massive-SCC)

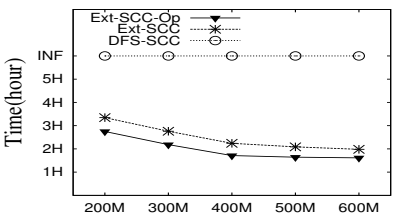

(c) Time (Large-SCC)

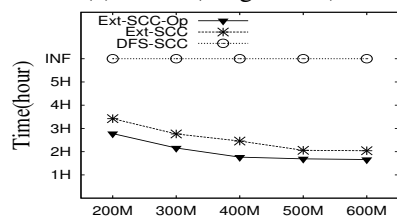

(e) Time (Small-SCC)

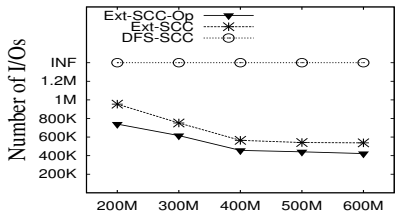

(b) I/Os (Massive-SCC)

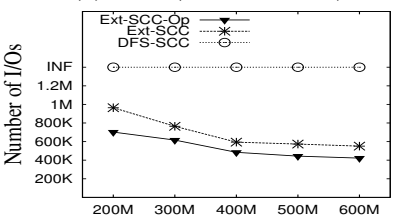

(d) I/Os (Large-SCC)

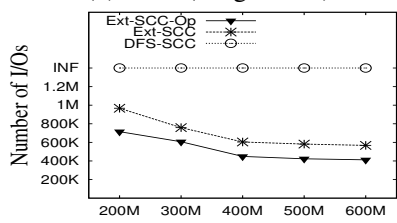

(f) I/Os (Small-SCC)
Fig. 8. Synthetic Data: Vary Memory Size

outperforms Ext-SCC in all cases. When the memory increases from $800 M$ to $1 G$, the costs for both Ext-SCC and Ext-SCCOp decrease sharply. The reason is that, in order to process the graph using Semi-SCC, $105,895,908 \times 8+256 K=847.4 M$ memory is needed, thus when the memory size is $1 G$, no iteration is needed and Semi-SCC can be directly applied on the original graph to output all SCCs.

Exp-2 (Vary Memory Size $M$ in Synthetic Data): To test the synthetic data, we vary the memory size $M$ from $200 M$ to $600 \mathrm{M}$. The time and I/O costs on Massive-SCC dataset are shown in Fig. 8(a) and Fig. 8(b) respectively. DFS-SCC cannot stop in limited time in all cases. Similar to the results on the real dataset in Fig. 7, when $M$ increases, the time and I/O costs for both Ext-SCC and Ext-SCC-Op decrease. When $M$ is smaller, the decrease rate is larger. This is because when $M$ is smaller, more iterations are needed for both Ext-SCC and ExtSCC-Op, and in the graph contraction phase, the contraction rate decreases when the number of iterations increases, since the graph becomes denser with larger number of iterations. Ext-SCC-Op outperforms Ext-SCC by $20 \%$ on average for both time and I/O consumptions. Fig. 8(c) and Fig. 8(d) show the results on Large-SCC dataset, and Fig. 8(e) and Fig. 8(f) show the results on Small-SCC dataset. The results for both Large-SCC and Small-SCC datasets are similar to those in the Massive-SCC dataset, and this is true for all the remaining test cases when varying other parameters in synthetic data. In the following, due to the lack of space, we only show the test results on the Large-SCC dataset.

Exp-3 (Vary Node Size $|V|$ in Synthetic Data): We vary the node size $|V|$ from $25 M$ to $200 M$, and the time and I/O costs are shown in Fig. 9(a) and Fig. 9(b) respectively. When $|V|$ increases, the time and I/O consumptions for both Ext-SCC and Ext-SCC-Op increase. This is because the stop condition for graph contraction is harder to be satisfied when $|V|$ is larger, and the cost on each iteration to scan and

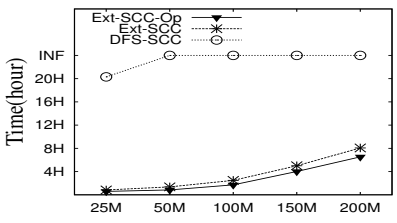

(a) Time (Vary $|V|)$

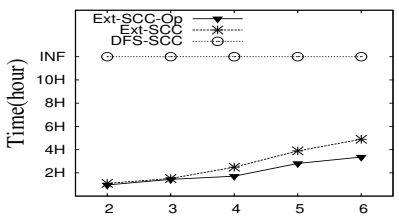

(c) Time (Vary Degree)

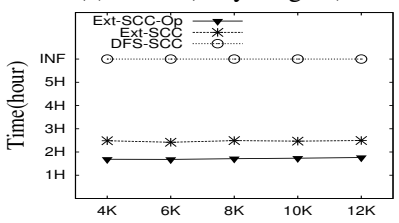

(e) Time (Vary SCC Size)

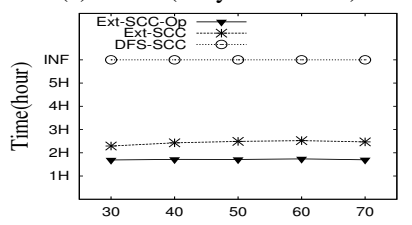

(g) Time (Vary SCC Num)

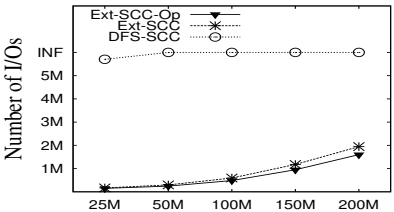

(b) I/Os (Vary $|V|$ )

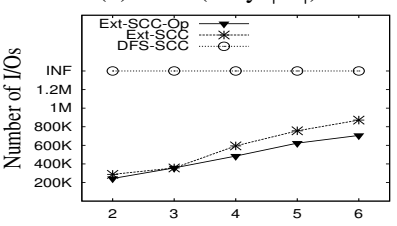

(d) I/Os (Vary Degree)

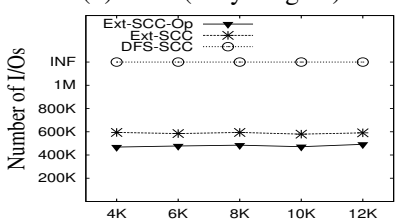

(f) I/Os (Vary SCC Size)

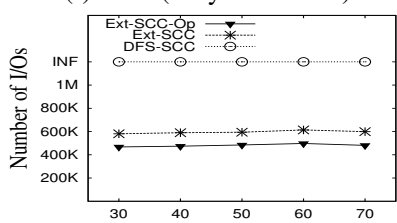

(h) I/Os (Vary SCC Num)
Fig. 9. Synthetic Data (Large-SCC)

sort nodes/edges is larger when $|V|$ is larger. Ext-SCC-Op outperforms Ext-SCC in all test cases. DFS-SCC cannot stop within the time limit when $|V| \geq 50 M$. When $|V|=25 M$, DFS-SCC consumes more than 20 hours while both Ext-SCC and Ext-SCC-Op consume less than 1 hour.

Exp-4 (Vary Average Degree in Synthetic Data): We vary the average degree $D$ of nodes from 2 to 6 . The time and I/O costs on Large-SCC are shown in Fig. 9(c) and Fig. 9(d) respectively. When $D$ increases, the time and I/O consumptions for both Ext-SCC and Ext-SCC-Op increase. This is because when $D$ increases, the number of edges increases. As a result, more iterations are needed and larger cost is consumed in each iteration as analyzed in Exp-1 when varying the graph size. Ext-SCC-Op outperforms Ext-SCC, and when $D$ is larger, the gap between Ext-SCC-Op and Ext-SCC is larger. This is because when number of edges is larger, more edges can be pruned by the edge reduction techniques used in Ext-SCC-Op.

Exp-5 (Vary SCC Size and SCC Number in Synthetic Data): Fig. 9(e) and Fig. 9(f) show the time and I/O costs when varying the average SCC size from $4 K$ to $12 K$ respectively. Fig. 9(g) and Fig. 9(h) show the time and I/O costs when varying the number of SCCs from 30 to 70 . When either the average SCC size increases, or the number of SCC $s$ increases, the time and I/O costs for both Ext-SCC and Ext-SCC-Op are not influenced much. As analyzed in Section VII, the key factors that influence the cost of Ext-SCC are the number of nodes and the number of edges of the graph. As a result, the size of SCC $s$ and the number of SCC $s$ do not have significant impact on the efficiency of our algorithms as long as $|E(G)|$ 
and $|V(G)|$ are fixed. This also explains why the results in the three datasets Massive-SCC, Large-SCC, and Small-SCC are similar as stated in Exp-2.

\section{IX, RELATED WORK}

Finding strongly connected components of a directed graph $G$ is a primitive operation in directed graph exploration, which has been studied for both internal memory model and external memory model. In the internal memory model, strongly connected components of a directed graph can be computed in $O(|V(G)|+|E(G)|)$ time based on DFS [12].

A naive way to externalize the internal DFS algorithm requires $O(|E|)$ I/Os. Chiang et al. [10] propose an algorithm with I/O complexity $O\left(|V|+\frac{|V|}{M} \cdot \operatorname{scan}(|E|)+\operatorname{sort}(|E|)\right)$. Later, Kumar and Schwabe [17] and Buchsbaum et al. [8] improve the I/O complexity to $O\left(\left(|V|+\frac{|E|}{B}\right) \log _{2} \frac{|V|}{B}+\operatorname{sort}(|E|)\right)$ by maintaining the list of nodes that should not be traversed using tournament trees [17] and buffered repository trees [8], respectively. Despite their theoretical guarantees, these algorithms are considered impractical for general directed graphs that encountered in real applications. Cosgaya-Lozano and Zeh [13] present a contraction based algorithm which contracts SCCs repeatedly until the graph fits in memory, then an internal memory algorithm is used to find the final SCCs. Such an algorithm may end up an infinite loop and cannot compute all SCCs. Both DFS based algorithm [8] and contraction based algorithm [13] are introduced in details in Section III.

In addition to external algorithms, there are semi-external algorithms for SCC computation which assume that all nodes of the graph can fit in the main memory. Sibeyn et al. [23] propose a semi-external DFS, which can be used to find all SCCs of a graph. Zhang et al. [26] improve such an algorithm by constructing and maintaining a special in-memory spanning tree of the graph. The semi-external algorithms [23] and [26] are introduced in details in Section III.

Other than the problem of finding SCCs or DFS tree on external directed graphs, several problems in the external memory model are studied in the literature. Dementiev et al. [14] provide an implementation of an external memory minimum spanning tree algorithm based on the ideas of [22], which performs extremely well in practice, even though theoretically inferior to the algorithms of [1], [10]. Ajwani et al. [4], [6] propose implementations of external undirected breadth-first search algorithm with the idea from [18]. Ulrich Meyer et al. [20], [21], [19] design and implement practical I/O-efficient single source shortest paths algorithm on general undirected sparse graphs. Surveys about designing I/O efficient algorithms for massive graphs can be found at [24], [5].

\section{$\mathrm{X}$. COnClusions}

In this paper, we study I/O efficient algorithms to find all SCCs for a directed graph, with the assumption that the nodes of the graph cannot reside entirely in memory. We overcome the deficiencies of the existing external SCC computation algorithms, and propose a new two-phase algorithm with graph contraction followed by graph expansion. We analyze the I/O cost of our approach and show that our algorithm can significantly reduce the number of random I/Os. We propose techniques to further reduce the I/O cost of our algorithm and confirm the I/O efficiency of our approaches using extensive experiments on both real and synthetic web scale graphs.

ACKNOWLEDGEMENTS: The work was supported by grant of the Research Grants Council of the Hong Kong SAR, China No. 418512.

\section{REFERENCES}

[1] J. Abello, A. L. Buchsbaum, and J. Westbrook. A functional approach to external graph algorithms. Algorithmica, 32(3), 2002.

[2] A. Aggarwal and J. S. Vitter. The input/output complexity of sorting and related problems. Commun. ACM, 31(9), 1988.

[3] A. V. Aho, J. E. Hopcroft, and J. D. Ullman. Data Structures and Algorithms. Addison-Wesley, 1983.

[4] D. Ajwani, R. Dementiev, and U. Meyer. A computational study of external-memory bfs algorithms. In Proc. of SODA'06, 2006.

[5] D. Ajwani and U. Meyer. Algorithmics of Large and Complex Networks, chapter 1: Design and Engineering of External Memory Traversal Algorithms for General Graphs. Springer, 2009.

[6] D. Ajwani, U. Meyer, and V. Osipov. Improved external memory bfs implementation. In Proc. of ALENEX'07, 2007.

[7] E. Angel, R. Campigotto, and C. Laforest. Analysis and comparison of three algorithms for the vertex cover problem on large graphs with low memory capacities. Algorithmic Operations Research, 6(1):56-67, 2011.

[8] A. L. Buchsbaum, M. H. Goldwasser, S. Venkatasubramanian, and J. Westbrook. On external memory graph traversal. In Proc. of SODA'00, 2000.

[9] J. Cheng, Y. Ke, S. Chu, and C. Cheng. Efficient processing of distance queries in large graphs: a vertex cover approach. In Proc. of SIGMOD'12, pages 457-468, 2012.

[10] Y.-J. Chiang, M. T. Goodrich, E. F. Grove, R. Tamassia, D. E. Vengroff, and J. S. Vitter. External-memory graph algorithms. In Proc. of SODA'95, 1995.

[11] N. Chiba and T. Nishizeki. Arboricity and subgraph listing algorithms. SIAM J. Comput., 14(1):210-223, 1985.

[12] T. H. Cormen, C. Stein, R. L. Rivest, and C. E. Leiserson. Introduction to Algorithms. McGraw-Hill, 2001.

[13] A. Cosgaya-Lozano and N. Zeh. A heuristic strong connectivity algorithm for large graphs. In Proc. of SEA'09, 2009.

[14] R. Dementiev, P. Sanders, D. Schultes, and J. F. Sibeyn. Engineering an external memory minimum spanning tree algorithm. In IFIP TCS, 2004

[15] W. Fan, J. Li, S. Ma, H. Wang, and Y. Wu. Graph homomorphism revisited for graph matching. PVLDB, 3(1), 2010.

[16] J. Hellings, G. H. Fletcher, and H. Haverkort. Efficient external-memory bisimulation on dags. In Proc. of SIGMOD'12, 2012.

[17] V. Kumar and E. J. Schwabe. Improved algorithms and data structures for solving graph problems in external memory. In Proc of SPDP'96, 1996.

[18] K. Mehlhorn and U. Meyer. External-memory breadth-first search with sublinear i/o. In Proc. of ESA'02, 2002.

[19] U. Meyer and V. Osipov. Design and implementation of a practical i/o-efficient shortest paths algorithm. In Proc. of ALENEX'09, 2009.

[20] U. Meyer and N. Zeh. I/O-efficient undirected shortest paths. In Proc. of ESA'03, 2003.

[21] U. Meyer and N. Zeh. I/O-efficient undirected shortest paths with unbounded edge lengths. In Proc. of ESA'06, 2006.

[22] J. F. Sibeyn. External connected components. In Proc. of SWAT'04, 2004.

[23] J. F. Sibeyn, J. Abello, and U. Meyer. Heuristics for semi-external depth first search on directed graphs. In Proc. of SPAA'02, 2002.

[24] J. S. Vitter. External memory algorithms and data structures. ACM Comput. Surv., 33(2), 2001.

[25] H. Yildirim, V. Chaoji, and M. J. Zaki. Grail: Scalable reachability index for large graphs. PVLDB, 3(1), 2010.

[26] Z. Zhang, J. X. Yu, L. Qin, L. Chang, and X. Lin. I/O efficient: Computing sccs in massive graphs. In Proc. of SIGMOD'13, 2013. 